\title{
In vitro interactions of Alternaria mycotoxins, an emerging class of food contaminants, with the gut microbiota: a bidirectional relationship
}

\author{
Francesco Crudo ${ }^{1,2}$. Georg Aichinger ${ }^{1} \cdot$ Jovana Mihajlovic $^{3} \cdot$ Elisabeth Varga $^{1} \cdot$ Luca Dellafiora $^{2} \cdot$ Benedikt Warth $^{1}$. \\ Chiara Dall'Asta ${ }^{2} \cdot$ David Berry $^{1,3,4} \cdot$ Doris Marko $^{1,2}$ (D)
}

Received: 21 December 2020 / Accepted: 1 April 2021 / Published online: 13 April 2021

(c) The Author(s) 2021

\begin{abstract}
The human gut microbiota plays an important role in the maintenance of human health. Factors able to modify its composition might predispose the host to the development of pathologies. Among the various xenobiotics introduced through the diet, Alternaria mycotoxins are speculated to represent a threat for human health. However, limited data are currently available about the bidirectional relation between gut microbiota and Alternaria mycotoxins. In the present work, we investigated the in vitro effects of different concentrations of a complex extract of Alternaria mycotoxins (CE; containing eleven mycotoxins; e.g. $0.153 \mu \mathrm{M}$ alternariol and $2.3 \mu \mathrm{M}$ altersetin, at the maximum CE concentration tested) on human gut bacterial strains, as well as the ability of the latter to metabolize or adsorb these compounds. Results from the minimum inhibitory concentration assay showed the scarce ability of CE to inhibit the growth of the tested strains. However, the growth kinetics of most of the strains were negatively affected by exposure to the various CE concentrations, mainly at the highest dose $(50 \mu \mathrm{g} / \mathrm{mL})$. The CE was also found to antagonize the formation of biofilms, already at concentrations of $0.5 \mu \mathrm{g} / \mathrm{mL}$. LC-MS/MS data analysis of the mycotoxin concentrations found in bacterial pellets and supernatants after $24 \mathrm{~h}$ incubation showed the ability of bacterial strains to adsorb some Alternaria mycotoxins, especially the key toxins alternariol, alternariol monomethyl ether, and altersetin. The tendency of these mycotoxins to accumulate within bacterial pellets, especially in those of Gram-negative strains, was found to be directly related to their lipophilicity.
\end{abstract}

Keywords Microbiome $\cdot$ Chemical mixture $\cdot$ Lipophilicity $\cdot$ Adsorption $\cdot$ Biofilm

$\begin{array}{ll}\text { Abbreviations } \\ \text { AOH } & \text { Alternariol } \\ \text { AME } & \text { Alternariol monomethyl ether } \\ \text { ALT } & \text { Altenuene } \\ \text { TeA } & \text { Tenuazonic acid } \\ \text { TEN } & \text { Tentoxin }\end{array}$

Doris Marko

doris.marko@univie.ac.at

1 Department of Food Chemistry and Toxicology, University of Vienna, Währinger Str. 38, 1090 Wien, Austria

2 Department of Food and Drug, University of Parma, Parco Area delle Scienze 27/A, 43124 Parma, Italy

3 Department of Microbiology and Ecosystem Science, Centre for Microbiology and Environmental Systems Science, University of Vienna, Althanstr. 14, 1090 Vienna, Austria

4 Joint Microbiome Facility of the Medical University of Vienna and the University of Vienna, Vienna, Austria

\author{
ALP Alterperylenol \\ AST Altersetin \\ ATX Altertoxin \\ STTX-III Stemphyltoxin III \\ CE Complex extract of Alternaria mycotoxins
}

\section{Introduction}

Through its metabolic activities and interactions with the host, the complex microbial community that inhabits the human intestine, termed the "gut microbiota", plays a key role in the well-being of humans (Clemente et al. 2012; Jandhyala et al. 2015). Any factor able to modify the composition and activity of the gut microbiota might influence both normal physiology and disease susceptibility of its host (Hasan and Yang 2019). Among the various factors influencing the gut microbial composition, diet is the most important one (Scott et al. 2013). In addition to the nutritional composition 
of ingested foods, the presence of food contaminants, such as mycotoxins, can affect the balance within the intestinal microbiome (Liew and Mohd-Redzwan 2018).

Mycotoxins are fungal toxic metabolites that may enter the food chain due to the ability of toxigenic fungi to infest a wide variety of crops and food commodities (Berthiller et al. 2013; Drejer Storm et al. 2014). Out of the roughly 400 mycotoxins known to date, only a few are regulated (Righetti et al. 2016). Among the compounds currently under consideration for new regulatory limits in the European Union, toxins produced by the genus Alternaria are gaining increasing interest due to the high level of occurrence in fresh and processed foods (e.g., fruits, vegetables, cereals, nuts, seeds and oils) and because of the toxic effects observed in vivo (e.g., teratogenic and fetotoxic effects) and in vitro (e.g., androgenic, estrogenic, genotoxic, mutagenic, and clastogenic effects) (Crudo et al. 2019). Alternaria species can produce more than 70 toxins, among which, alternariol (AOH), alternariol monomethyl ether (AME), altenuene (ALT), tenuazonic acid (TeA), tentoxin (TEN), alterperylenol/alteichin (ALP), and the altertoxins (ATXs) I and II, are the best characterized (Crudo et al. 2019). Of note, the dibenzo- $\alpha$-pyrones AOH and AME, and the epoxide-carrying compounds ATX-II and stemphyltoxin III (STTX-III) were reported to induce DNA strand breaks in vitro and to act as topoisomerase poisons and topoisomerase inhibitors, respectively (Fehr et al. 2009; Fleck et al. 2016). AOH and AME also showed clastogenic, mutagenic and estrogenic effects (Brugger et al. 2006; Lehmann et al. 2006; Dellafiora et al. 2018), as well as the ability to modulate innate immunity (Grover and Lawrence 2017; Kollarova et al. 2018). Information about the absorption, distribution and excretion of Alternaria toxins is currently available only in mice (Schuchardt et al. 2017) and rats (Puntscher et al. 2019a), while it is still scarce in humans. Consequently, the real impact on human health has yet to be clarified. A recent in vivo study in rats showed a fecal excretion $>89 \%$ for $\mathrm{AOH}$ and AME, while the perylene quinones ATX-I and ALP were recovered only at very low levels $(<5 \%)$ (Puntscher et al. 2019a). Interestingly, the highly reactive epoxide-carrying compounds ATX-II and stemphyltoxin III (STTX-III) were not recovered at all (Puntscher et al. 2019a). On this basis, the high level of fecal excretion found for $\mathrm{AOH}$ and $\mathrm{AME}$ suggests that potential toxic effects are likely to occur predominantly at the level of the intestinal tract rather than systemically. Thus, possible inhibitory or growth-promoting effects on members of the gut microbiota cannot be excluded. On the other hand, the low recovery of ATX-I, ATX-II, STTXIII, and ALP might be the consequence of chemical transformation occurring at the gastro-intestinal level. Considering the ability of the human gut microbiota to participate in the detoxification processes of xenobiotic compounds (Collins and Patterson 2020), the involvement of intestinal bacteria in the mechanisms of removal of ingested mycotoxins must be hypothesized, especially in view of a possible risk assessment of Alternaria mycotoxins.

A bi-directional interaction between mycotoxins and the gut microbiota has been reported by several authors, who investigated the effects exerted by mycotoxins on the gut microbiota and the ability of gut microbes to bind or metabolize mycotoxins ingested with the diet. As an example, germ-free rats inoculated with fecal microbiota from healthy humans showed a significant decrease of the concentration levels of Escherichia coli and an increase of the Bacteroides/Prevotella group after treatment with the Fusarium mycotoxin deoxynivalenol (DON) (Saint-Cyr et al. 2013). On the other hand, the ability of microorganisms from the intestines of broilers to transform DON to the less toxic de-epoxy-DON was also reported (He et al. 1992; Yu et al. 2010). Another mycotoxin that was shown to perturb the gut bacterial population is T2-toxin, which led to an increase of the aerobic bacteria in the intestines of rats and swine (Tenk et al. 1982). Microorganisms from human feces were found to release the parental compound from the glucoside metabolites of T2-toxin, thus contributing to the increase of the amount of free absorbable mycotoxin (Gratz et al. 2017). The transformation of the mycotoxin zearalenone (ZEN) into unknown metabolites (Gratz et al. 2017), as well as its ability to modify the microbial diversity in porcine ascending colon contents, has also been reported (Piotrowska et al. 2014). A modulation of microbiota composition was also found to be induced by ochratoxin A and aflatoxin B1 (Baines et al. 2013; Ouethrani et al. 2013; Guo et al. 2014; Wang et al. 2016), as well as by mixtures of fumonisins B1 + B2 (Burel et al. 2013) and ZEN + DON (Piotrowska et al. 2014).

We recently summarized the available data on the cooccurrence of Alternaria mycotoxins in food, highlighting the high frequency of co-occurrence of multiple metabolites (Crudo et al. 2019). Based on this, a complex extract of Alternaria alternata cultured on rice, containing eleven chemically characterized mycotoxins, was used in the present work to investigate the bidirectional relation existing between Alternaria mycotoxins and the gut microbiota. The aim of this work was to evaluate the effects exerted by the complex mixture of Alternaria mycotoxins on human gut bacterial strains belonging to five different phyla. To investigate the possible contribution of bacteria to the reduction of their harmful effects, the ability of the same bacterial strains to metabolize or adsorb Alternaria mycotoxins was studied.

\section{Materials and methods}

\section{Complex extract of Alternaria mycotoxins (CE)}

The mycotoxin extract used in the present study is a complex mixture of Alternaria mycotoxins obtained in our laboratory 
by growing the Alternaria alternata strain DSM 62,010 on long rice with a subsequent extraction procedure, as previously described (Puntscher et al. 2019b). The obtained extract was chemically characterized by LC-MS/MS. Mycotoxin concentrations in the extract are listed in Table 1 and adapted from Aichinger et al. (2019). The chemical structures of mycotoxins contained in the extract are shown in Online Resource 1.

\section{Chemicals for sample preparation and LC-MS/MS analysis}

Methanol and acetonitrile were acquired from Honeywell (Seelze, Germany), water was purchased from VWR International GmbH (Vienna, Austria), while ammonium acetate and $25 \%$ ammonia solution in water were obtained from Sigma-Aldrich Handels GmbH (Vienna, Austria). All solvents were of LC-MS grade. Reference materials of Alternaria toxins were kindly provided by other researchers or purchased from several suppliers. For further details, refer to Puntscher et al. (2019b).

\section{Bacterial strains and culture media}

The human gut bacterial strains were acquired from culture collections or isolated from human feces in our laboratory and identified through the 16S rRNA gene sequencing. Detailed information about the strains and media used in this study can be found in Online Resource 2. Fourteen bacterial strains were chosen to have at least one representative strain for each of the most dominant gut microbial phyla (i.e. Firmicutes, Bacteroidetes, Actinobacteria, Proteobacteria, and Verrucomicrobia) and to test both Gram-negative and Grampositive strains. Bacteroides caccae (BC), Bacteroides eggerthii (BE), Bacteroides thetaiotaomicron (BT), Bacteroides

Table 1 Composition of the Alternaria mycotoxin extract characterized by LC-MS/MS analysis (adapted from Aichinger et al. 2019)

\begin{tabular}{lc}
\hline Alternaria mycotoxins (Abbreviations) & $\begin{array}{l}\text { Concentration } \\
\text { (mg toxin/g } \\
\text { extract) }\end{array}$ \\
\hline Alternariol (AOH) & 0.79 \\
Alternariol monomethyl ether (AME) & 0.65 \\
Altenuene (ALT) & 0.78 \\
Tenuazonic acid (TEA) & 597 \\
Tentoxin (TEN) & 0.02 \\
Altertoxin I (ATX-I) & 9.92 \\
Altertoxin II (ATX-II) & 14.1 \\
Alterperylenol (ALP) & 12.6 \\
Stemphyltoxin III (STTX-III) & 21.0 \\
Altenusin (ALS) & 0.28 \\
Altersetin (AST) & 18.4 \\
\hline
\end{tabular}

vulgatus (BV), Parabacteroides distasonis (PD), Escherichia coli (EC), and Clostridium innocuum (CI), were cultivated in a supplemented brain heart infusion broth (BHI-S, ATCC Medium 1293), while for Alistipes finegoldii (AF), Alistipes timonensis (AT), and Ruminococcus bicirculans $(R B)$ a modified YCFA broth (Yeast extract-Casein hydrolysate-fatty acids) supplemented with glucose was employed (YCFAg, DSMZ Medium 1611). For the growth of Akkermansia muciniphila, brain hearth infusion broth (Oxoid; CM1135) supplemented with $0.05 \%$ pig gastric mucin type II (SigmaAldrich, M2378) was prepared (BHI-muc). A modified MRS broth (De Man, Rogosa, Sharpe; m-MRS) was used for the growth of Lactobacillus hominis, Bifidobacterium longum and Bifidobacterium sp. The m-MRS broth was composed as follows (per liter): $100 \mathrm{~g}$ Tryptone (Oxoid; LP0042), $10 \mathrm{~g}$ beef extract (Sigma-Aldrich; B4888), $5 \mathrm{~g}$ yeast extract (Oxoid; LP0021), 20 g glucose (Oxoid; LP0071), $1 \mathrm{~g}$ tween 80 (Fluka; 93,780), $2 \mathrm{~g} \mathrm{~K}_{2} \mathrm{HPO}_{4}$ (Merck; 1.05104.1000), $5 \mathrm{~g}$ Na-acetate (Sigma-Aldrich; S8750), $0.2 \mathrm{~g} \mathrm{MgSO}_{4}$ (SigmaAldrich; M2643). Prior to performing an experiment, media were left at least for $20 \mathrm{~h}$ in the anaerobic tent $\left(5 \% \mathrm{CO}_{2}, 5 \%\right.$ $\mathrm{H}_{2}$ and $90 \% \mathrm{~N}_{2}$ ) to allow deoxygenation.

\section{Determination of the minimum inhibitory concentration (MIC)}

Determination of the MIC values of the Alternaria mycotoxin extract was preliminarily performed on $B$. thetaiotaomicron, B. vulgatus, B. caccae, C. innocuum and E. coli with the aim to investigate the ability of the extract to completely inhibit the growth of the strains and define the concentrations to be tested for the subsequent analysis. Briefly, strains were grown overnight in BHI-S medium under anaerobic conditions. Then, the optical density at $600 \mathrm{~nm}\left(\mathrm{OD}_{600}\right)$ was set to 0.1 and $50 \mu \mathrm{l}$ of the adjusted cultures were pipetted in 96-well plates and diluted with $50 \mu \mathrm{l}$ of the test media containing the various concentrations $(2 \times$ concentrated) of the $\mathrm{CE}$ dissolved in DMSO. Thus, the final $\mathrm{OD}_{600}$ was 0.05 . For each CE concentration tested, wells containing the corresponding percentages of DMSO were also prepared as controls. The 96-well plates also included growth controls (media plus bacteria, without DMSO) and a negative control (media). The final CE concentrations tested were 200, $100,50,25,12.5,6.25,3.13,1.56 \mu \mathrm{g} / \mathrm{mL}$ (maximum DMSO concentration of $0.4 \%$ ) and each concentration was tested in three biological replicates. Plates were incubated under anaerobic and static conditions for $24 \mathrm{~h}$ at $37{ }^{\circ} \mathrm{C}$, followed by optical density measurements of the wells at $600 \mathrm{~nm}$ with a microplate reader (Multiskan ${ }^{\mathrm{TM}}$ GO Microplate Spectrophotometer, Thermo Scientific). The lowest concentration of CE which reduced bacterial growth by $90 \%$ or higher was considered to be the MIC value. 


\section{Analysis of the growth curves}

Determination of bacterial growth curves of the strains was carried out by measuring the optical density at $600 \mathrm{~nm}$. Each strain was grown overnight in its appropriate medium at $37{ }^{\circ} \mathrm{C}$ under anaerobic conditions. Afterward, the overnight culture was diluted with the appropriate medium to a starting $\mathrm{OD}_{600}$ of 0.1 , dispensed into wells of a sterile 96-well flatbottomed microtiter plate (Costar 3595, Corning Inc., Corning, NY, USA) and diluted 1:1 with the test media containing the different concentrations of CE or DMSO (final volume of $200 \mu \mathrm{l} /$ well). The final $\mathrm{CE}$ and DMSO concentrations tested were $50 \mu \mathrm{g} / \mathrm{mL}, 25 \mu \mathrm{g} / \mathrm{mL}, 5 \mu \mathrm{g} / \mathrm{mL}, 0.5 \mu \mathrm{g} / \mathrm{mL}$, and $0.1 \%, 0.05 \%, 0.01 \%, 0.001 \%$, respectively. The final $\mathrm{OD}_{600}$ was 0.05 . The prepared plates, which also included negative controls (media) and growth controls (media plus bacteria, without DMSO), were wrapped with parafilm to prevent dehydration and incubated for $24 \mathrm{~h}$ at $37{ }^{\circ} \mathrm{C}$ in a microplate reader (Multiskan ${ }^{\mathrm{TM}}$ GO Microplate Spectrophotometer, Thermo Scientific) placed in an anaerobic tent. Plates were shaken continuously at low speed ( $5 \mathrm{~Hz}$, amplitude $15 \mathrm{~mm}$ ) during the incubation time and for $10 \mathrm{~s}$ at medium speed ( $11 \mathrm{~Hz}$, amplitude $3 \mathrm{~mm}$ ) prior to each read. The $\mathrm{OD}_{600}$ was recorded automatically every $20 \mathrm{~min}$ for a total of $24 \mathrm{~h}$. Each condition was tested in at least three biological replicates. Quantification of the area under the experimental growth curves was carried out using the $\mathrm{R}$ package Growthcurver, available for installation from the Comprehensive R Archive Network (CRAN).

\section{Quantification of biofilm biomasses}

Evaluation of the ability of the extract to affect the formation of biofilms produced by E. coli, B. vulgatus, B. thetaiotaomicron, and B. caccae was carried out according to the microtiter-plate test described by Stepanović et al. (2007) with slight modifications. Briefly, overnight cultures of the selected strains were diluted with their strain-specific media to an $\mathrm{OD}_{600}$ of 0.1 . Then, $75 \mu \mathrm{l}$ of the adjusted cultures was transferred into 96-well cell culture plates and diluted 1:1 with the test media containing the different percentages of DMSO (final concentrations of $0.1 \%, 0.05 \%, 0.01 \%$, $0.001 \%$ ) or concentrations of $\mathrm{CE}$ (final concentrations of $50 \mu \mathrm{g} / \mathrm{mL}, 25 \mu \mathrm{g} / \mathrm{mL}, 5 \mu \mathrm{g} / \mathrm{mL}, 0.5 \mu \mathrm{g} / \mathrm{mL})$. The final $\mathrm{OD}_{600}$ was 0.05 . Plates were incubated for $24 \mathrm{~h}$ or $48 \mathrm{~h}$ under anaerobic conditions and without shaking. Afterward, $\mathrm{OD}_{600}$ values were recorded to exclude bacterial growth inhibitions (Haney et al. 2018), plates were emptied, washed twice with tap water to remove planktonic cells and heat-fixed at $60{ }^{\circ} \mathrm{C}$ for $1 \mathrm{~h}$. Biofilms were stained by adding $200 \mu \mathrm{l}$ of $1 \%$ crystal violet solution to each well and incubating the plates at room temperature (RT) for $15 \mathrm{~min}$. Then, plates were emptied, washed tree times with tap water and dried overnight at
RT. Crystal violet was resolubilized by adding $200 \mu \mathrm{l} 30 \%$ acetic acid. After $15 \mathrm{~min}$ incubation at RT, absorbance at $570 \mathrm{~nm}$ was measured by means of a microtiter plate reader (Multiskan $^{\mathrm{TM}}$ GO Microplate Spectrophotometer, Thermo Scientific). Conditions for each strain were tested in at least three biological replicates. Definition of cut-off values, that allowed to distinguish biofilm-producing from non-biofilmproducing strains, was performed according to Stepanović et al. (2007) as follows: OD $_{\text {cut-off }}=$ mean ODs of uninoculated medium +3 times standard deviation.

\section{Sample preparation for LC-MS/MS analysis}

After $24 \mathrm{~h}$ incubation of the strains with the various concentrations of $\mathrm{CE}$, the bacterial suspension of each well was collected and immediately stored at $-80{ }^{\circ} \mathrm{C}$. To investigate whether the bacterial strains were able to metabolize or adsorb mycotoxins of the Alternaria extract, samples treated with $25 \mu \mathrm{g} / \mathrm{mL}$ of $\mathrm{CE}$ were chosen to be analyzed by LC-MS/MS analysis. The choice to analyze samples from this concentration was made by evaluating both the detection limits of mycotoxins and the results obtained through the analysis of the bacterial growth curves. The aim was to analyze the samples whose CE concentration had caused only a minor or no effect on bacterial growth, in such a way as not to affect the metabolic activity of the bacteria tested. For the extraction of mycotoxins from supernatants, treated and control samples were thawed and centrifuged at 20,000 ref for $10 \mathrm{~min}\left(4^{\circ} \mathrm{C}\right)$ to pellet the bacteria. Then, the supernatants were transferred into new tubes, diluted 1:5 with an ice-cold extraction solvent (ACN/MeOH, 1:1, v/v), vortexed and placed at $-20{ }^{\circ} \mathrm{C}$ for $1 \mathrm{~h}$ to allow precipitation of proteins. Then, samples were centrifuged (20,000 rcf, $10 \mathrm{~min}$, $4{ }^{\circ} \mathrm{C}$ ) and the resulting supernatants were transferred into HPLC vials. As for the extraction from the bacterial pellets, after pelleting the bacteria through centrifugation $(20,000$ rcf, $10 \mathrm{~min}, 4^{\circ} \mathrm{C}$ ), a step of washing with phosphate buffer saline solution (PBS, $0.1 \mathrm{~mol} / \mathrm{L}, \mathrm{pH} 7.4$ ) was performed to ensure the removal of mycotoxins present in the tube and deriving from the removed supernatants. After the PBS was removed, the washed bacterial pellets were resuspended with an extraction solvent (ACN/MeOH/water, 2:2:1, v/v/v), vortexed and sonicated for $15 \mathrm{~min}$ (on ice). After $1 \mathrm{~h}$ incubation at $-20{ }^{\circ} \mathrm{C}$, precipitated proteins and bacteria were pelleted by centrifugation $\left(20,000 \mathrm{rcf}, 10 \mathrm{~min}, 4^{\circ} \mathrm{C}\right.$ ) and supernatants transferred into HPLC vials. Extracted samples were immediately analyzed or stored at $-80^{\circ} \mathrm{C}$ until analysis.

\section{LC-MS/MS analysis}

The extracted pellets, supernatants, and controls were analyzed using a high-performance liquid chromatographic system (UltiMate3000, Dionex Thermo Fisher Scientific) 
coupled to a TSQ Vantage triple quadrupole mass spectrometer equipped with a heated electrospray ionization interface (Thermo Fisher Scientific).

The LC-MS/MS method used in the present study has been already used in our recent work for the quantification of Alternaria mycotoxins in human fecal slurries (Crudo et al. 2020). Briefly, a Supelco Ascentis ${ }^{\circledR}$ Express C18 column $(100 \times 2.1 \mathrm{~mm}, 2.7 \mu \mathrm{m})$ equipped with a pre-column (SecurityGuard $^{\mathrm{TM}}, \mathrm{C} 18,2 \mathrm{~mm}$, Phenomenex, Torrance, CA, USA) was employed for the chromatographic separation. An ammonium acetate in water solution $(5 \mathrm{mM}, \mathrm{pH}$ adjusted to 8.7 with a $25 \% \mathrm{NH}_{4} \mathrm{OH}$ solution) and $\mathrm{MeOH}$ were used as eluents. MS data for the extracted samples were acquired in multiple reaction monitoring mode, applying negative electrospray ionization. Further information about the applied LC-MS/MS method can be found in Puntscher et al. (2018). External calibration was employed as a quantification technique and injection of the calibration set was performed after every 20-30 samples. Monitoring of the instrumental conditions and the data acquisition were carried out using the software package Thermo Xcalibur ${ }^{\mathrm{TM}}$ (v. 4.0.27.42, Thermo Scientific), while TraceFinder ${ }^{\mathrm{TM}}$ software (v. 3.3; Thermo Scientific) was employed for data evaluation.

\section{In silico prediction of mycotoxins lipophilicity}

The prediction of mycotoxins lipophilicity was performed using the freely available online tool SwissADME (http:// www.swissadme.ch). The "consensus $\log P_{\mathrm{o} / \mathrm{w}}$ value" for each mycotoxin, which is the arithmetic mean of the values obtained by five computational methods, was used for the LC-MS/MS data evaluation.

\section{Statistical analysis}

Significant differences $(p<0.05$ or $p<0.01)$ between control and treated samples were evaluated by Independent Student $t$ test, performed using SPSS software (v. 23.0, SPSS Inc., Chicago, IL, USA). Principal component analysis of the LC-MS/MS data was carried out using OriginPro software (v. 2018, OriginLab Corporation, Northampton, MA, USA).

\section{Results}

\section{Effects of Alternaria mycotoxins on bacterial strains}

\section{Minimum inhibitory concentration (MIC)}

The complex Alternaria extract (CE), containing eleven characterized mycotoxins (Table 1), was tested against the human gut species Bacteroides thetaiotaomicron, Bacteroides vulgatus, Bacteroides caccae, Escherichia coli, and
Clostridium innocuum to evaluate its ability to inhibit the growth of the strains. Results from the test showed the inability of the extract to inhibit the growth of $B$. thetaiotaomicron, B. caccae, and E. coli at any tested concentration of the Alternaria extract (ranging from 1.56 to $200 \mu \mathrm{g} / \mathrm{mL}$ ). In contrast, MIC values of $50 \mu \mathrm{g} / \mathrm{mL}$ and $100 \mu \mathrm{g} / \mathrm{mL}$ were found for B. vulgatus and C. innocuum, respectively.

\section{Influence on bacterial growth kinetics}

The ability of the Alternaria extract to affect the growth kinetics of 14 reference human gut bacterial strains was evaluated by monitoring the optical density of microbial suspensions treated with $0.5,5,25$, and $50 \mu \mathrm{g} / \mathrm{mL}$ of $\mathrm{CE}$ for $24 \mathrm{~h}$. Bacterial growth curves of all fourteen strains treated with the various concentrations of CE are shown in Online Resource 3. As summarized in Fig. 1, while increased or decreased of area under the curve (AUC) were found for most of the bacterial strains after exposure to specific $\mathrm{CE}$ concentrations (regardless of the Gram-type), the growth of some strains was not affected even after treatment with the highest $\mathrm{CE}$ concentration. Examples of the most diverse behaviors observed after exposure to the maximum and minimum concentrations of $\mathrm{CE}$ are reported in Fig. 2, where the growth curves of Alistipes finegoldii (2A, 2B), B. thetaiotaomicron (2C, 2D), B. vulgatus (2E, 2F), E. coli $(2 \mathrm{G}, 2 \mathrm{H})$, and Ruminococcus bicirculans (2I, 2J) are shown.

Among all the strains tested, B. vulgatus was the most sensitive to the effects of $\mathrm{CE}$, with its growth being

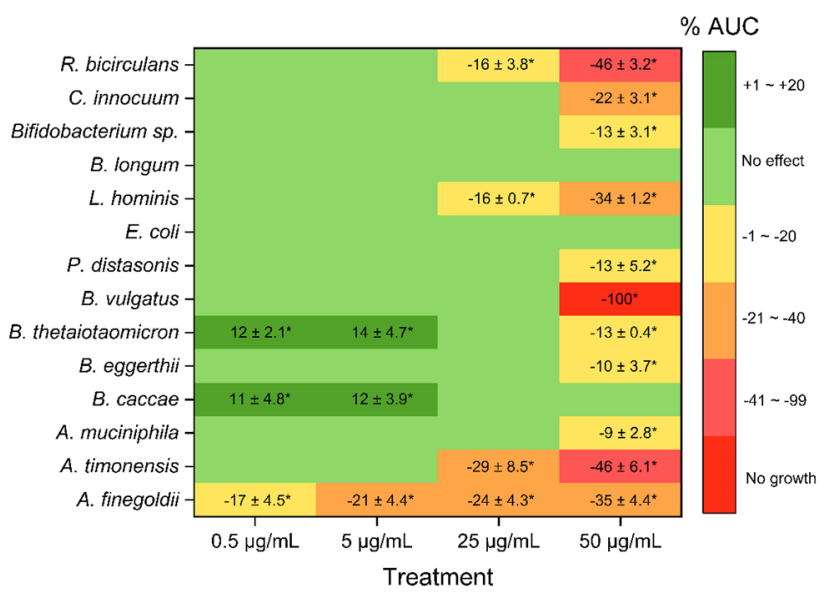

Fig. 1 Modifications of the area under the curve (AUC) of growth curves induced by $24 \mathrm{~h}$ incubation of bacterial strains with different concentrations of the Alternaria extract. Reported numbers refer to mean $\pm \mathrm{SD}$ of increase or reduction of AUC compared to the respective control (strain +DMSO). Percentages of DMSO varied from $0.1 \%$ to $0.001 \%$ (for treatments with $50 \mu \mathrm{g} / \mathrm{mL}$ or $0.5 \mu \mathrm{g} / \mathrm{mL}$ of CE, respectively). Each condition was tested in triplicate. Significant differences to the DMSO control were evaluated by Student's $t$ test $(* p<0.05)$ 
completely suppressed at $50 \mu \mathrm{g} / \mathrm{mL}$. Differently, the other tested strains belonging to the genus Bacteroides, namely $B$. eggerthii, B. thetaiotaomicron, and B. caccae, were only slightly or not at all affected by an incubation with the maximum concentration of the CE (Figs. 1, 2a). Treatments of B. thetaiotaomicron and B. caccae with 5 and $0.5 \mu \mathrm{g} / \mathrm{mL}$ of CE led to an increase of the AUC values. Although no complete inhibition of the growth was detected after treatment of A. finegoldii (in-house isolated strain) with any of the $\mathrm{CE}$ concentrations tested, its growth was negatively affected by treatment with all CE concentrations. The growth of Akkermansia muciniphila and Parabacteroides distasonis (in-house isolated strain) was only slightly affected by treatment with the highest $\mathrm{CE}$ concentration, while E. coli was found to be the least sensitive Gram-negative strain to the Alternaria extract, as none of the incubations resulted in a change of growth kinetics. With regard to the growth of Gram-positive bacteria, none of the strains was affected by a CE-exposure to 0.5 and $5 \mu \mathrm{g} / \mathrm{mL}$. Except for the type-strain of Bifidobacterium longum, all Gram-positive strains were affected at $50 \mu \mathrm{g} / \mathrm{mL}$ CE. A reduction of AUC values by $16 \pm 0.7 \%$ and $16 \pm 3.8 \%$ was also detected after treatment of $L$. hominis and R. bicirculans (in-house isolated strain) with $25 \mu \mathrm{g} / \mathrm{mL}$ of CE. As shown in Online Resource 3, most of the detected AUC reductions were a consequence of the reduction of the stationary-phase growth yield, without
Fig. 2 Representative growth curves expressed as optical density measured at $600 \mathrm{~nm}$ of different bacterial strains during treatment with the maximum (50 $\mu \mathrm{g} / \mathrm{mL}$, left column) and minimum $(0.5 \mu \mathrm{g} / \mathrm{mL}$, right column) concentration of the Alternaria extract (CE). Bacterial growth curves colored in black or red refer to the DMSOtreated or CE-treated strains, respectively

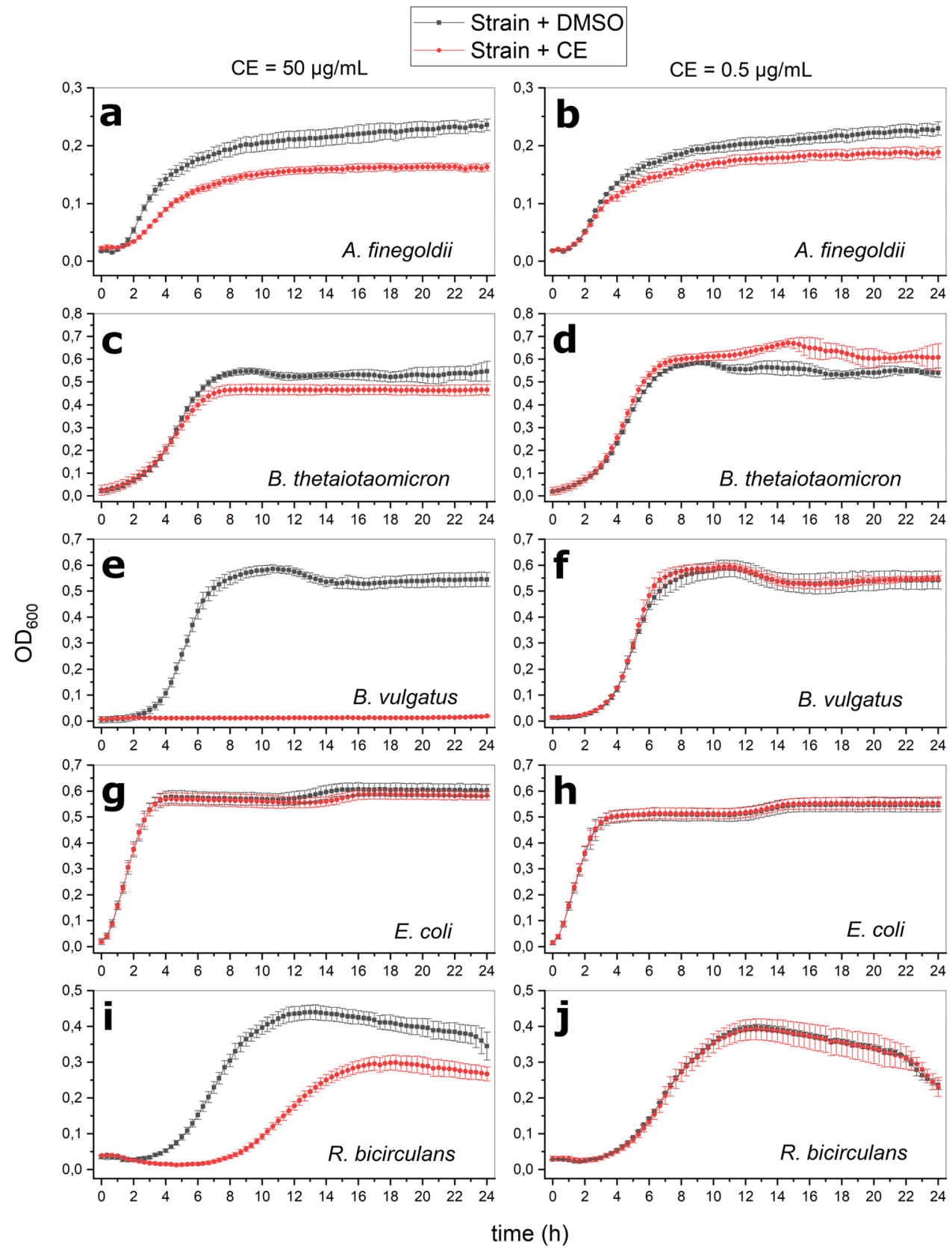


any modification of the length of the lag phase. However, a reduction of the AUC caused by an extension of the lag phase together with a decreased growth yield was instead detected after treatment of $R$. bicirculans with the highest concentration of CE tested (Fig. 2i). Of note, exposure of $A$. timonensis, L. hominis, and Bifidobacterium sp. to $50 \mu \mathrm{g} / \mathrm{mL}$ of CE, as well as of $A$. finegoldii to $50 \mu \mathrm{g} / \mathrm{mL}, 25 \mu \mathrm{g} / \mathrm{mL}$ and $5 \mu \mathrm{g} / \mathrm{mL}$ of CE resulted in increased values of doubling time (Online Resource 4).

\section{Influence of Alternaria extract on biofilm production}

The ability of the extract to affect biofilm production of gut bacteria was evaluated by employing the strains $E$. coli, $B$. vulgatus, B. thetaiotaomicron, and B. caccae. Except for the treatment of $B$. vulgatus with the maximum concentration of CE tested, all observed modifications in biofilm biomasses were attributed to the direct inhibition of biofilm formation by the $\mathrm{CE}$, and not to a reduction of general bacterial growth, as confirmed through the analysis of the growth curves and the measurement of the $\mathrm{OD}_{600}$ values during each biofilm assay (Online Resource 5). As shown in Fig. 3, a concentration-dependent reduction of the biofilm biomass produced by $E$. coli was detected only after $48 \mathrm{~h}$ incubation of the strain with all $\mathrm{CE}$ concentrations (maximum reduction of $59 \pm 10 \%$ for treatments with $50 \mu \mathrm{g} / \mathrm{mL}$ ). Similar results were obtained for $B$. vulgatus, whose biofilm-forming capacity was not affected by $24 \mathrm{~h}$ incubation with the various $\mathrm{CE}$ concentrations, except for the treatment with the highest concentration. However, the absence of biofilm mass at this concentration cannot be traced back to a direct effect of the extract on the biofilm-forming capacity of the strain but to a growth inhibition, as previously reported and showed in Online Resource 5. After $48 \mathrm{~h}$ incubation, treatments of B. vulgatus with $25 \mu \mathrm{g} / \mathrm{mL}, 5 \mu \mathrm{g} / \mathrm{mL}$, and $0.5 \mu \mathrm{g} / \mathrm{mL}$ led to reductions of the biofilm biomasses. The highest level of reduction $(40 \pm 2 \%)$ was found for the treatment with the lowest $\mathrm{CE}$ concentration. Exposure of $B$. thetaiotaomicron and $B$. caccae to the various concentrations of $\mathrm{CE}$ led to reductions of the biofilm biomasses after $24 \mathrm{~h}$. However, these effects were not persistent, as no difference between treated and control samples was found after $48 \mathrm{~h}$ incubation. Interestingly, treatment of $B$. thetaiotaomicron with the highest $\mathrm{CE}$ concentration did not result in any modification of biofilm production, while the treatment with $5 \mu \mathrm{g} / \mathrm{mL}$ led to the most pronounced reduction in biofilm biomass $(48 \pm 8 \%)$. Similar results were obtained for $B$. caccae, whose strongest biomass reduction was reached after treatment with $5 \mu \mathrm{g} /$ $\mathrm{mL}$ of CE (24 h). However, treatment with $50 \mu \mathrm{g} / \mathrm{mL}$ of CE resulted in a reduction of the biofilm biomass of $36 \pm 14 \%$.

\section{Effects of bacterial strains on Alternaria mycotoxins}

\section{Total recovery of Alternaria mycotoxins}

Quantification of mycotoxins in bacterial suspensions treated with $25 \mu \mathrm{g} / \mathrm{mL}$ of $\mathrm{CE}$, carried out by liquid chromatography tandem-mass spectrometry (LC-MS/MS), revealed that among the mycotoxins originally present in the extract, the highly reactive epoxide-carrying Alternaria toxins STTXIII and ATX-II, were found neither in bacteria-containing samples nor in the control media after $24 \mathrm{~h}$. For this reason, they were excluded from the evaluation of the effects induced by the bacterial strains tested on the mycotoxins of the extract. The Alternaria mycotoxins AOH, AME, ALP, ALS, and AST were found to be the most affected ones in terms of total recovery (Fig. 4; Online Resource 6). Significant losses of $\mathrm{AOH}(p<0.05)$, ranging from $20 \pm 3 \%$ to $67 \pm 4 \%$ (P. distasonis and E. coli, respectively), occurred with all tested strains, except for B. eggerthii, Bifidobacterium $s p$. (in-house isolated strain) and $R$. bicirculans. The recovery of AME, which chemically differs from $\mathrm{AOH}$ by an additional methyl group, was slightly higher. As for the mycotoxin ALP, the highest levels of recovery were obtained for samples incubated with the lactic acid bacteria (LAB) L. hominis, B. longum and Bifidobacterium sp., while very low levels of recovery were obtained for the other strains. Interestingly, samples containing the strain of Bifidobacterium $s p$. were characterized by higher amounts of ALP (+17 $\pm 2 \%)$ compared to the control medium. An opposite trend was found in the total recovery of the Alternaria mycotoxin ALS, whose concentrations were significantly lower $(p<0.05)$ only in the LAB strains. A total recovery of AST was not obtained after incubation of the extract with most of the strains tested. ALT, TeA, TEN, and ATX-I were the mycotoxins less affected by the incubation with the various strains (Online Resources 6 and 7), since no significant difference between mycotoxin concentrations in media controls and in strain-containing samples was found for most of the strains after $24 \mathrm{~h}$ incubation. Notably, significant increased ATX-I concentrations $(p<0.05)$ by $42 \pm 1 \%, 83 \pm 4 \%$, and $36 \pm 6 \%$ were found in samples incubated with B. eggerthii, P. distasonis, and Bifidobacterium sp., respectively.

\section{Distribution of mycotoxins in pellets and supernatants of the tested strains and influence of mycotoxins lipophilicity}

LC-MS/MS analysis of the mycotoxin concentrations found in bacterial pellets and supernatants showed a different tendency of mycotoxins to bind to bacterial cells or to remain in solution (Fig. 4; Online Resource 7). Figure 5a shows the average amount of mycotoxins found in pellets of all strains tested after $24 \mathrm{~h}$ incubation with $25 \mu \mathrm{g} / \mathrm{mL}$ of CE compared 


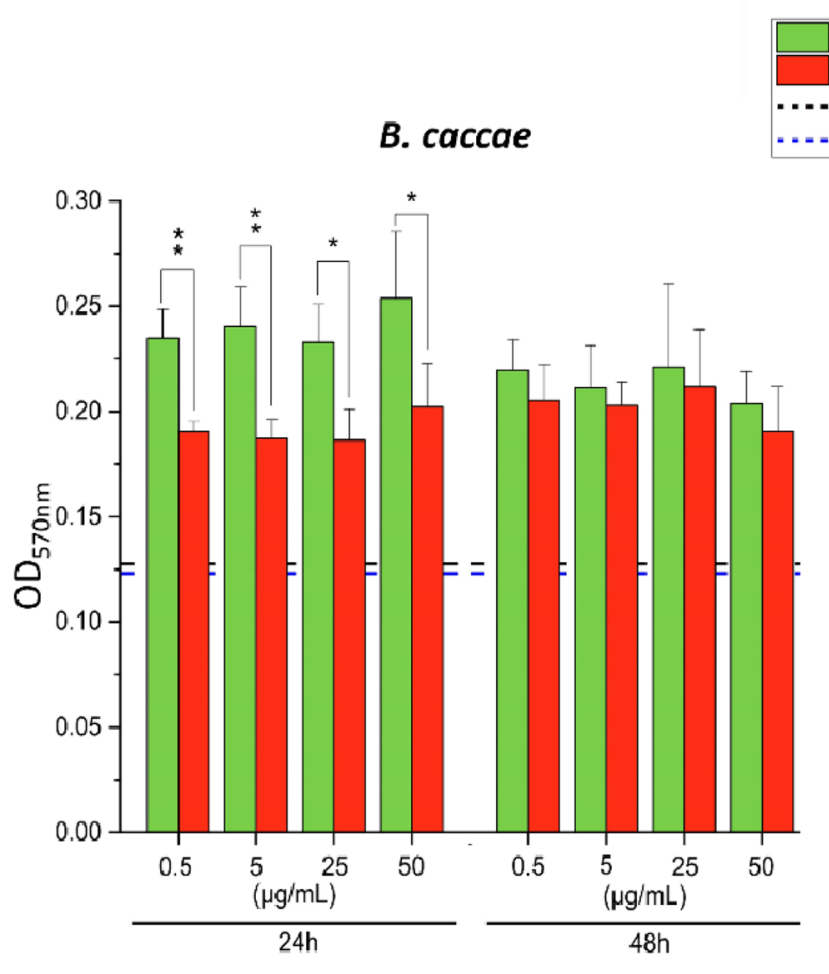

\section{B. vulgatus}

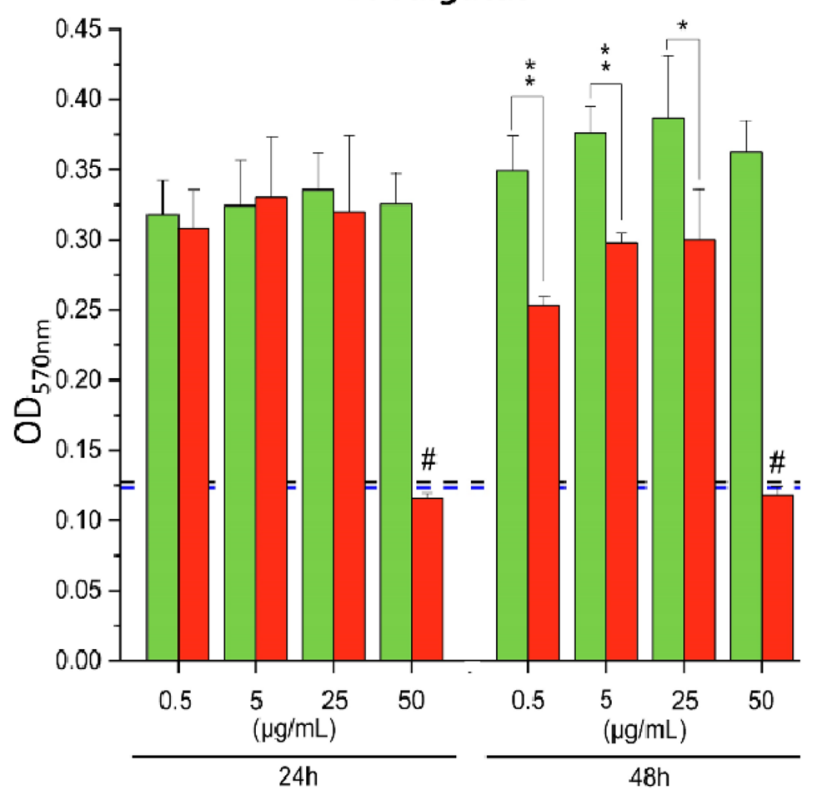

Fig. 3 Effects on biofilm formation induced by $24 \mathrm{~h}$ and $48 \mathrm{~h}$ incubation of strains with different concentrations of the Alternaria extract. Differences between treated and control samples were evaluated by

to the total amount recovered (pellet + supernatant). The mycotoxins ALT, TeA, TEN, and ALS were only found in supernatants of the tested strains, while the other mycotoxins were also found in bacterial pellets. The perylene quinone ATX-I and the mycotoxin ALP were present only at very
DMSO

CE

- cut-off $24 \mathrm{~h}$

$=$ - cut-off $48 \mathrm{~h}$

B. thetaiotaomicron

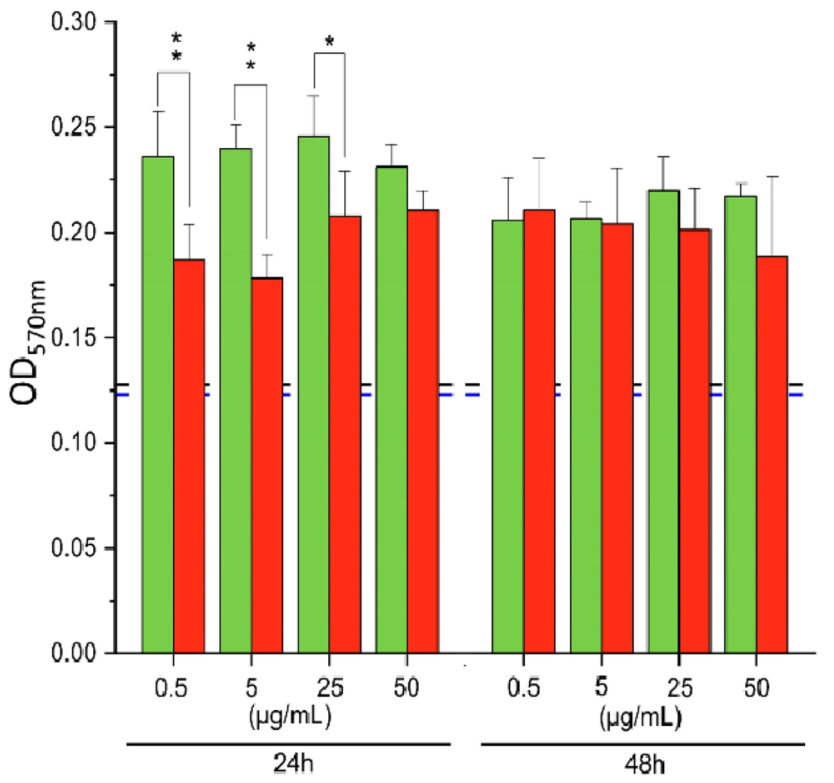

E. coli

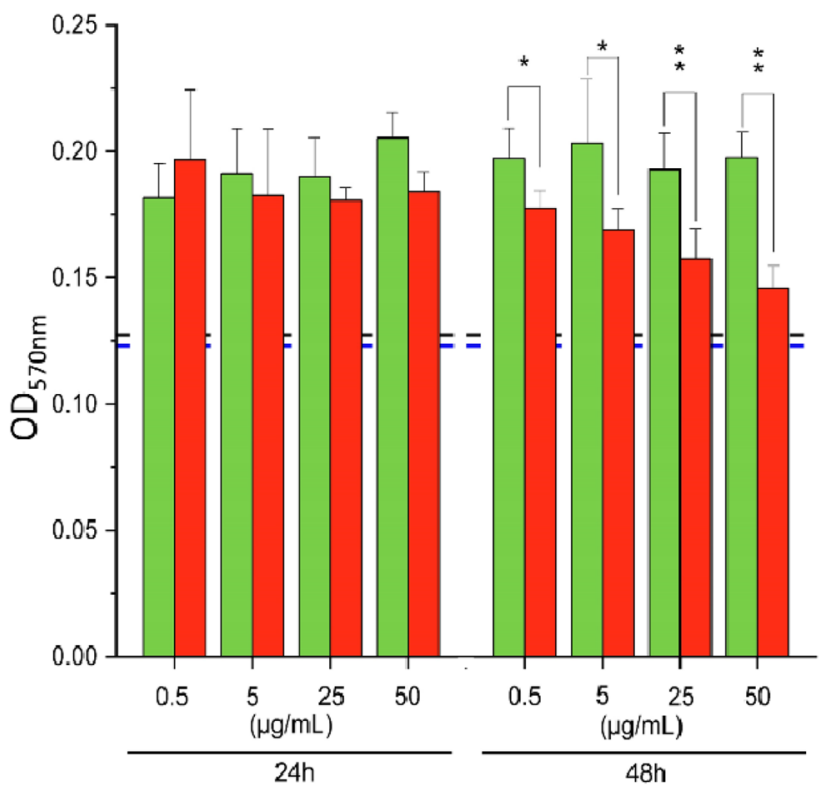

Student's $t$ test $(* p<0.05 ; * * p<0.01)$. Cut-off values were calculated as follow: $\mathrm{OD}_{\text {cut-off }}=$ mean ODs of uninoculated medium +3 times standard deviation. "Indicates no growth

low concentrations in pellets of the bacterial strains tested and no difference between their concentrations in pellets of Gram-positive and Gram-negative strains was observed. Among the Alternaria mycotoxins of the extract, a rather marked tendency of adsorption of AOH, AME, and AST by 

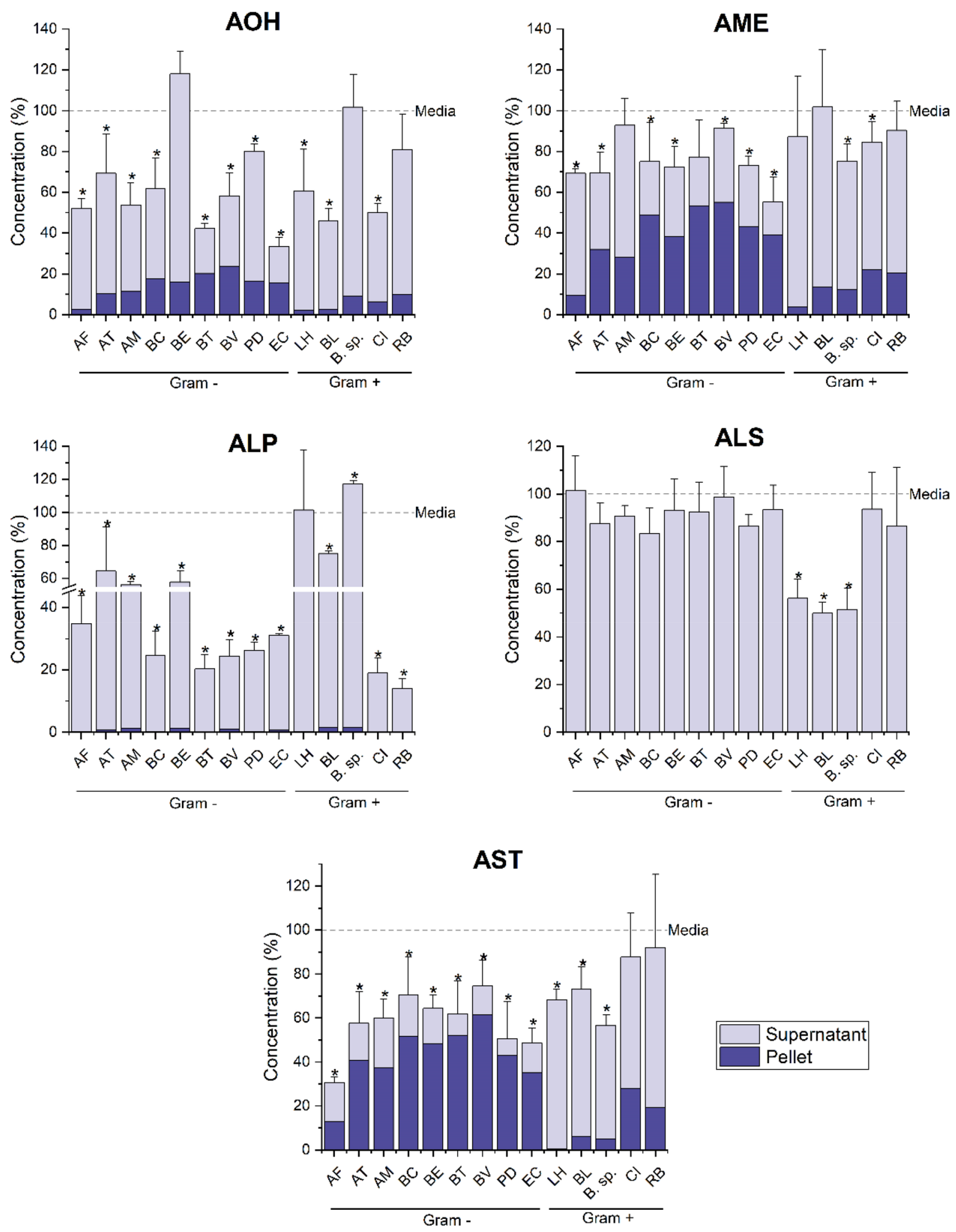

Fig. 4 Bar charts showing the amount of the most affected mycotoxins recovered in pellets and supernatants of the tested strains after $24 \mathrm{~h}$ incubation with $25 \mu \mathrm{g} / \mathrm{mL}$ of the Alternaria extract. Data are reported as mean $\pm \mathrm{SD}$ and differences between the total mycotoxin recovery in samples and media controls (media $+\mathrm{CE}$ ) were evaluated by Student's $t$ test $\left({ }^{*} p<0.05\right)$. AF A. finegoldii, AT A. timonensis, AM A. muciniphila, $B C B$. caccae, BE B. eggerthii, BT B. thetaiotaomicron, $B V$ B. vulgatus, $P D$ P. distasonis, EC E. coli, $L H$ L. hominis, $B L$ B. longum, B. sp. Bifidobacterium sp., CI C. innocuum, RB R. bicirculans 
a

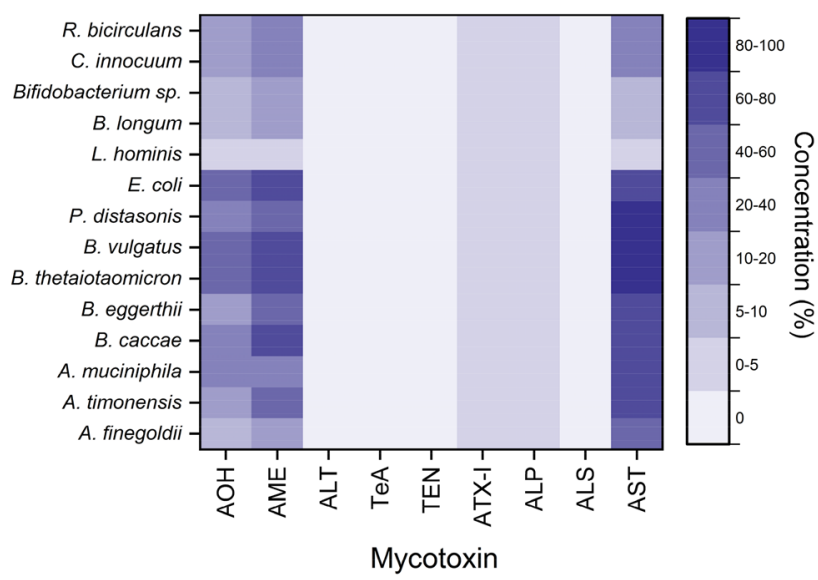

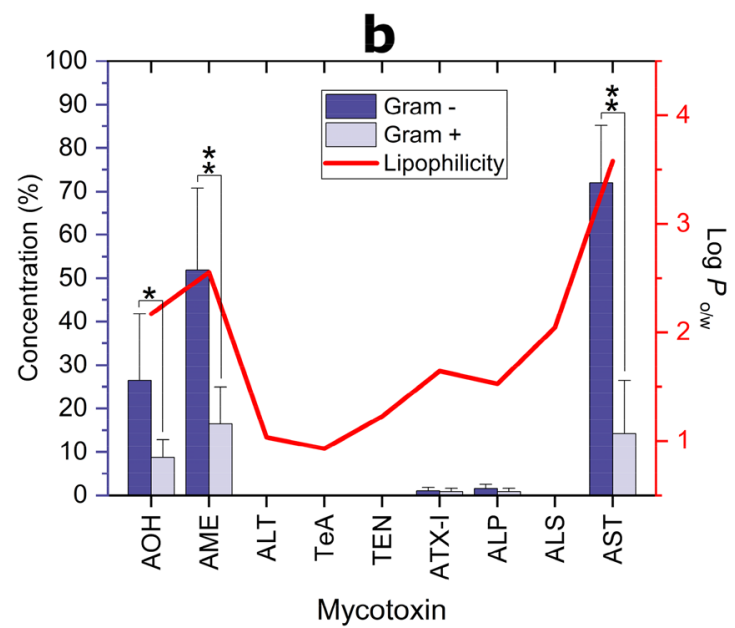

Fig. 5 Recoveries of mycotoxins from bacterial pellets. a Heatmap showing the average amount (in \% compared to the total amount recovered) of mycotoxins found after $24 \mathrm{~h}$ incubation with $25 \mu \mathrm{g} /$ $\mathrm{mL}$ of $\mathrm{CE}$ in pellets of all strains tested. b Double-axis plot showing mean $\pm \mathrm{SD}$ (in \% compared to the total amount recovered) of myco-

the various strains was observed (Fig. 5a). The mean concentrations of $\mathrm{AOH}$ found in pellets of Gram-negative strains were significantly higher $(26 \pm 15 \% ; p<0.05)$ than in pellets of Gram-positive strains $(8.7 \pm 4.1 \%)$, as shown in Fig. $5 b$. Similarly, AME concentrations in pellets of Gram-negative bacteria $(52 \pm 19 \%)$ were significantly different $(p<0.01)$ from those found in Gram-positive strains $(16 \pm 9 \%)$. The Alternaria mycotoxin showing the highest level of accumulation in bacterial pellets was found to be AST, whose mean recovery levels from pellets were higher $(p<0.01)$ for Gram-negative $(72 \pm 13 \%)$ than for Gram-positive bacteria $(14 \pm 12 \%)$. To avoid a possible misinterpretation of data related to mycotoxin concentrations in bacterial pellets due to the different OD600 values characterizing the bacterial suspensions, the experimentally determined mycotoxin concentrations were also normalized based on a theoretical OD600 value of 0.5 (Online Resource 8). As shown, data confirmed the tendency of mycotoxins to accumulate preferentially in the pellets of Gram-negative bacteria. The accumulation of $\mathrm{AOH}, \mathrm{AME}$, and AST within the Gram-negative bacterial pellets was a key factor contributing to the separation of Gram-positive from Gram-negative strains along the principal component (PC) 1 in the principal component analysis (PCA, Fig. 6). Gram-negative and Gram-positive bacteria distributed along the positive and negative axes of the PC1, respectively. However, four bacterial strains (A. finegoldii, A. muciniphila, $R$. bicirculans, $C$. innocuum) were overlapped and distributed along the negative axis of the $\mathrm{PC} 2$, thus showing mycotoxin distribution patterns similar to both Gram staining types. An in-depth investigation on the lipophilic properties of the Alternaria mycotoxins contained in the extract, performed using the SwissADME online tool, toxin concentrations found in pellets of Gram-negative and positive strains (blue and light blue columns, respectively; left axis), and the mean value of theoretical lipophilicity (solid line; right axis) of mycotoxins. Significant differences between Gram-negative and -positive strains were evaluated by Student's $t$ test $\left({ }^{*} p<0.05 ;{ }^{* *} p<0.01\right)$

revealed a direct correlation between the presence of mycotoxins in pellets and the in silico predicted values of lipophilicity of mycotoxins (Fig. 5b). The highest value for the n-octanol/water partition coefficient $\left(\log \mathrm{P}_{\mathrm{o} / \mathrm{w}}\right)$ was obtained for AST $(3.58 \pm 1.24)$, followed by AME $(2.55 \pm 0.62), \mathrm{AOH}$ $(2.17 \pm 0.60)$, ALS $(2.05 \pm 0.65)$, ATX-I $(1.64 \pm 0.77)$, and $\operatorname{ALP}(1.53 \pm 0.66)$. Although these results are in accordance with the mycotoxin concentrations found in pellets, ALP was not found in any pellet of the tested strains. Of note,

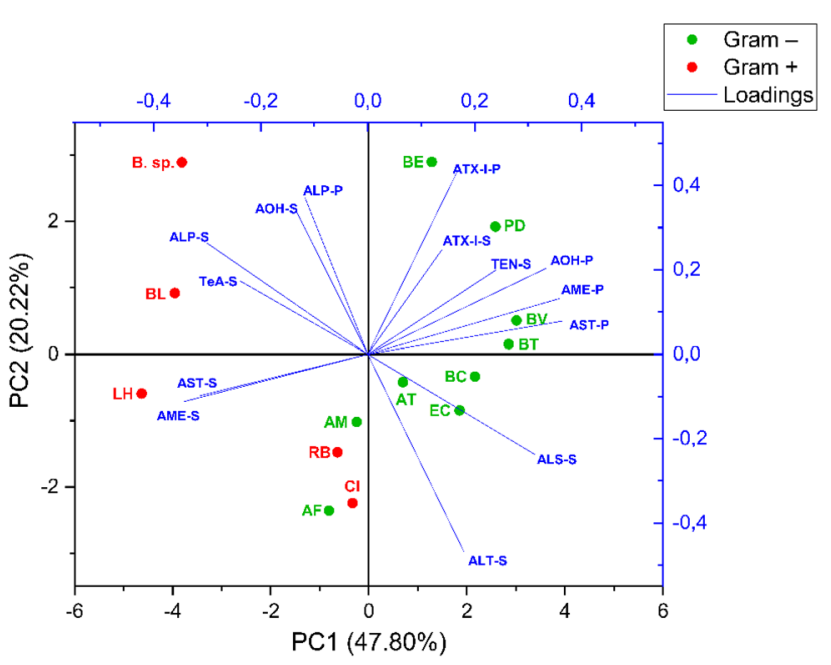

Fig. 6 Score-loading plot of the first two principal components from PCA highlighting strain distribution in the bidimensional space and the contribution of mycotoxin concentrations found in pellets and supernatants. AF A. finegoldii, AT A. timonensis, AM A. muciniphila, $B C B$. caccae, $B E$ B. eggerthii, $B T$ B. thetaiotaomicron, $B V B$. vulgatus, $P D$ P. distasonis, EC E. coli, LH L. hominis, BL B. longum, B. sp. Bifidobacterium sp., CI C. innocuum, RB R. bicirculans 
the mycotoxins not found in the pellets of the tested strains (i.e. ALT, TeA, and TEN) were characterized by the lowest $\log \mathrm{P}_{\mathrm{o} / \mathrm{w}}$ predicted levels $(1.03 \pm 0.51,0.93 \pm 0.76$, and $1.23 \pm 1.36$, respectively).

\section{Discussion}

A preliminary investigation examining the potential of the Alternaria extract to completely inhibit the growth of five selected strains (B. thetaiotaomicron, B. vulgatus, B. caccae, $C$. innocuum and E. coli) revealed no or low activity of the extract. Considerable MIC values were found only for B. vulgatus $(50 \mu \mathrm{g} / \mathrm{mL})$ and $C$. innocuum $(100 \mu \mathrm{g} / \mathrm{mL})$, while the other strains were not affected even at the maximum concentration of the CE $(200 \mu \mathrm{g} / \mathrm{mL})$. Despite this, most of the bacteria exhibited changes in their growth kinetics after exposure to specific concentrations of the extract (Fig. 1; Online Resource 3). A representative set of examples is reported in Fig. 2. In particular, while the AUC of $A$. finegoldii was reduced by the exposure to both maximum (Fig. 2a) and minimum (Fig. 2b) concentrations of CE, no changes in growth kinetics were found for E. coli (Fig. 2g, h) and the ATCC 15,697 strain of B. longum (Fig. 1), which were the most resistant bacteria against Alternaria toxins in study at hand. Strains belonging to the genus Bacteroides deserve particular attention because of the high intra-genus variability in their response to the presence of the Alternaria extract. Exposure of $B$. vulgatus to the maximum $\mathrm{CE}$ concentration resulted in growth inhibition (Fig. 2e), while exposure to the lowest $\mathrm{CE}$ concentration did not affect its growth (Fig. 2f). Exposure of B. eggerthii and B. thetaiotaomicron to the maximum $\mathrm{CE}$ concentration only slightly affected their growth, while no changes were found for $B$. caccae at this concentration. The strains $B$. thetaiotaomicron and $B$. caccae exhibited increased AUC values after exposure to the lowest $\mathrm{CE}$ concentrations tested $(5 \mu \mathrm{g} / \mathrm{mL}$ and $0.5 \mu \mathrm{g} / \mathrm{mL}$ ). This might be the consequence of a mycotoxininduced modulation of intra-cellular pathways involved in bacterial growth, or the result of a possible utilization of some of the Alternaria mycotoxins as carbon and energy sources. Supporting this interpretation, bacteria were previously reported to metabolize xenobiotics, and the demethylation of compounds can provide a carbon source for their growth (Koppel et al. 2017). However, considering the lack of data in the literature, further studies are required to better clarify this phenomenon. All the observed modifications in bacterial growth discussed above were found to be mainly attributable to modifications of the stationary-phase growth yield and, in some cases, increased doubling times were also observed (Online Resource 4), suggesting a manifold action on bacterial growth worth of being further analyzed. $R$. bicirculans was the only strain for which an extended duration of the lag phase could be observed after treatment with $50 \mu \mathrm{g} / \mathrm{mL}$ of CE (Fig. 2i). The prolongation of the lag phase can be considered a defense mechanism that allows bacteria to survive unfavorable growth conditions, such as presence of antimicrobial compounds. As an example, the growth of a clinical isolate of Enterococcus faecium was delayed by the treatment with the fluoroquinolones ciprofloxacin and moxifloxacin, which act by inhibiting the DNA gyrase (Theophel et al. 2014). Interestingly, some of the Alternaria mycotoxins contained in the extract (i.e. $\mathrm{AOH}$, AME, ATX-I, ATX-II, ALP, and STTX-III) were previously reported to target the bacterial gyrase (Jarolim et al. 2017). Consistently with the growth inhibitions and changes in the growth kinetics observed in the present study, the ability of Alternaria mycotoxins to exert antimicrobial effects was previously reported by several authors. AOH inhibited the growth of Staphylococcus aureus and Corynebacterium betae at micromolar concentrations, while the growth of an E. coli strain was only slightly reduced (Freeman 1966). AME and ALT were active against both Gram-positive and Gram-negative bacteria (Pero et al. 1971; Lou et al. 2016). The mycotoxin ALS exhibited antimicrobial activity against several multidrug-resistant bacteria (Kjer et al. 2009), while AST only inhibited the growth of pathogenic Gram-positive strains (MIC values ranging from 0.12 to $2 \mu \mathrm{g} / \mathrm{mL}$ ) (Hellwig et al. 2002). The concentrations of AST the strains tested in the present study were exposed to (see Online Resource 9) were in the MIC range reported above (Hellwig et al. 2002). Hence, this mycotoxin might have at least contributed not only to the changes of the growth kinetics of the Gram-positive bacteria, but also to the growth inhibition of C. innocuum (Gram-positive) after treatment with $100 \mu \mathrm{g} /$ $\mathrm{mL}$ of CE. However, considering that the other Alternaria mycotoxins of the extract were present in significantly lower concentrations (Online Resource 9) than those previously reported in literature to be able to exert antimicrobial effects (Freeman 1966; Pero et al. 1971; Kjer et al. 2009; Lou et al. 2016) and that the growth of Gram-negative bacteria was also influenced, the observed effects might be the consequence of synergistic effects caused by the co-presence of multiple Alternaria mycotoxins.

It is important to note that some of the mycotoxin concentrations tested could be reached in the GI tract upon consumption of contaminated food. In fact, assuming a complete bio-accessibility of mycotoxins, their dilution in a total volume of gastrointestinal fluids of $1.1 \mathrm{~L}$ (maximum volume of gastrointestinal fluids reported by Schiller et al. 2005), an intake of $315 \mathrm{~g}$ of sweet peppers, according to the maximum concentrations of mycotoxins found by Gambacorta et al. (2018), would be sufficient to exceed the concentrations of AOH and ALT tested in the present study. As for AME, TEN and TeA, less than $50 \mathrm{~g}$ of soya beans, $50 \mathrm{~g}$ of bread and $250 \mathrm{~g}$ of tomatoes would be sufficient according to the 
contamination reported by Oviedo et al. (2012), Zhao et al. (2015) and Stinson et al. (1981), respectively. Unfortunately, the shortage of occurrence and exposure data related to the other mycotoxins of the extract prevents a precise calculation. Anyway, based on this and the results obtained in the present work, further studies are necessary to clarify the actual occurrence of these mycotoxins in food on the one hand, and in vivo investigations aimed at evaluating the effects of Alternaria mycotoxins on the gut microbiome would be desirable on the other hand, considering the current lack of data and the possible negative consequences resulting from changes of its composition. Indeed, gut dysbiosis, characterized by a reduced or increased presence of specific bacteria or groups of bacteria, has been linked both to the development of pathologies related to digestive tract and other organ systems (Carding et al. 2015). As an example, a possible involvement of some pathobiont strains of $E$. coli in the pathogenesis of inflammatory bowel disease (IBD), which includes Crohn's disease (CD) and ulcerative colitis (UC), has been suggested (Perna et al. 2020). Patients with $C D$ were found to have a higher abundance of $E$. coli at the level of the ileum than healthy people (Perna et al. 2020). On the contrary, the abundance of A. muciniphila, which is one of the most important mucolytic symbionts inhabiting the human intestine, was found to be reduced in IBD, autism, and obesity (Derrien et al. 2017). A lower abundance of the genus Bacteroides, compared to healthy controls, was also reported in CD and UC patients in active phase (Zhou and Zhi 2016).

Although it has not yet been clarified whether these alterations in the abundance of intestinal bacteria are a cause or a consequence of IBD, the modifications in bacterial growth observed in the present work raise the question whether exposure to Alternaria toxins may actually represent a contributing factor to the development of diseases, such as IBD.

An in-depth investigation with selected strains (E. coli, B. vulgatus, B. thetaiotaomicron and B. caccae) revealed also the ability of the Alternaria extract to affect biofilm formation. As already known, microorganisms inhabiting the human intestinal tract live as complex biofilm communities in close association with the outer layer of the host mucus (Buret et al. 2019). The disruption of the complex structure of the biofilm, which confers microorganisms increased tolerance to stress, may lead not only to diseases at the gastrointestinal level, but also in other organs (Buret et al. 2019). In the present study, the biofilm production by $E$. coli was reduced in a concentration-dependent manner after $48 \mathrm{~h}$ incubation with all concentrations of $\mathrm{CE}$, without changes in the growth of the strain. Similar results were reported by Lee et al. (2014), who reported the ability of coumarin and eight derivatives to inhibit the formation of biofilms produced by a pathogenic $E$. coli strain. Of note, treatment with $50 \mu \mathrm{g} / \mathrm{mL}$ of ellagic acid (derivative), which is structurally related to some of the Alternaria mycotoxins (AOH, AME), led to the reduction of about $40 \%$ of the biofilm biomass produced by the strain (Lee et al. 2014). Although ellagic acid was not tested, coumarin and the two derivatives umbelliferone and esculetin were found to repress curli genes and motility genes. In addition, the observed reductions in fimbriae production and swarming motility were linked to the observed transcriptional modifications. On this basis, it cannot be excluded that, in the present study, some of the Alternaria mycotoxins might have triggered changes in the transcription of genes involved in biofilm formation. Unlike $E$. coli, the reduction in biofilm biomass observed for strains belonging to the genus Bacteroides did not follow a clear concentration-dependent pattern. In addition, while the biofilm production of $B$. vulgatus was affected after $48 \mathrm{~h}$ incubation, reduction of biomasses was found for B. caccae and B. thetaiotaomicron after $24 \mathrm{~h}$, but not after $48 \mathrm{~h}$. This suggests possible losses of the antibiofilm properties of the extract over time or the onset of adaptive cellular responses to mycotoxin-induced stress. The detailed elucidation of underlying mechanisms will be addressed in subsequent studies. Apart from the differences observed in the time of onset of inhibitory effects on biofilm production (i.e. $24 \mathrm{~h}$ or $48 \mathrm{~h}$ ), one of the most interesting results obtained from the analysis of data related to the production of biofilms was the most effective inhibition of biofilm formation observed in strains of the genus Bacteroides exposed to the lowest $\mathrm{CE}$ concentrations. Interestingly, similar results have been reported upon treatment of bacterial strains with both pure compounds (i.e. antibiotics; Majidpour et al. 2017) and complex mixtures (i.e. essential oils; Papa et al. 2020). Despite no information is currently available about both the mechanisms underlying this phenomenon and the anti- or pro-biofilm properties of the single compounds of the Alternaria extract, it could be hypothesized that some mycotoxins of the extract exert pro-biofilm effects, while others act as anti-biofilm factors, resulting, in mixture, in an inhibition of the biofilm production. However, at the lowest CE concentrations, the pro-biofilm mycotoxins may not be within their concentration range of biological activity to exert effects in the strains of the genus Bacteroides, thus they might not be able to counteract the anti-biofilm effects of the other mycotoxins of the extract.

To evaluate the ability of the tested strains to chemically modify Alternaria toxins, quantification of mycotoxins in bacterial pellets and supernatants was performed by LC-MS/ MS analysis. Results clearly showed no or low impact of bacterial strains on the total recovery of ALT, TeA, TEN, and ATX-I (Online Resource 7). An increased concentration of ATX-I after $24 \mathrm{~h}$ incubation was found in samples incubated with $B$. eggerthii, $P$. distasonis, and Bifidobacterium $s p$. compared to the controls without bacteria. These higher concentrations might be a consequence of de-epoxidation 
processes involving the highly reactive epoxide-carrying mycotoxin ATX-II. In fact, an in vivo study carried out by Puntscher et al. (2019a) reported the presence of ATX-I in plasma, urine and fecal samples of rats administered with the Alternaria mycotoxin ATX-II. This transformation was also reported in eukaryotic cell lines (Fleck et al. 2014a, b). Moreover, in a recent study (Crudo et al. 2020), we found higher concentrations of ATX-I in samples of fecal slurries of 3 out 4 donors after a $3 \mathrm{~h}$ anaerobic incubation with the same Alternaria extract used in the present study. The ability of gut microorganisms from broilers, pigs, and rats to transform the epoxide-carrying compounds DON into de-epoxyDON was also reported (Worrell et al. 1989; He et al. 1992; Kollarczik et al. 1994; Young et al. 2007). Although in the present study, ATX-II was found neither in samples containing bacteria nor in media controls after $24 \mathrm{~h}$ incubation, in our previous study, we reported ATX-II to be still present after $3 \mathrm{~h}$ incubation (Crudo et al. 2020). Thus, it is probable that the transformation of ATX-II into ATX-I mediated by B. eggerthii, $P$. distasonis, and Bifidobacterium sp. occurred in the first hours after incubation with the Alternaria extract.

The transformation of the epoxide-carrying Alternaria mycotoxin STTX-III into ALP was also reported in literature (Fleck et al. 2014a, b). In the present study, most of the bacteria mediated a reduction of the total content of ALP. Of note, ALP concentrations in samples incubated with Bifidobacterium sp. were higher than those present in the control media, suggesting a possible transformation of STTX-III to ALP by this bacterium. Among the other Alternaria mycotoxins, significant unrecovered amounts of $\mathrm{AOH}$, AME, and AST were found in samples incubated with most of the strains, without any difference between Gram staining types. The incomplete recovery of $\mathrm{AOH}$ was also reported by Lemke et al. (2016) who recovered percentages of the mycotoxin ranging from 70 to $85 \%$ from pure cultures of $E$. coli DH5 $\alpha$ and L. plantarum BFE5092. However, no data are currently available about the total recovery of the other mycotoxins after incubation with pure cultures, including ALS, for which we observed an incomplete recovery in samples from the three strains of LAB. The uncomplete recovery obtained for some mycotoxins might be a consequence of their metabolization by the bacterial strains tested. As a matter of fact, the gut microbiota has been already reported to modify the chemical structure of several compounds, including food components and contaminants (Koppel et al. 2017). To date, although no information is available about gut microbial metabolites of the Alternaria mycotoxins, several polyphenolic compounds, having chemical structures similar to some Alternaria mycotoxins, have been reported to be metabolized by the gut microbiome (Makarewicz et al. 2021). Considering that the profile of metabolites produced depends not only on the chemical structure of the original compound but also on the repertoire of enzymes expressed in the intestinal microbiome, which can vary on an individual basis (Koppel et al. 2017), it is difficult to hypothesize the chemical structure of possible metabolites of Alternaria mycotoxins. However, the mycotoxin $\mathrm{AOH}$ has a chemical structure very similar to urolithins, a class of gut microbial metabolites produced from ellagic acid and, among which, urolithin $\mathrm{C}$ has recently been reported to reduce the absorption and metabolism of $\mathrm{AOH}$ in a Caco-2 cell model exploiting its chemical analogies to AOH (Crudo et al. 2021). Of note, the production of the various urolithins occurs through successive removal of hydroxyl groups (Espín et al. 2013). Based on this, a loss of hydroxyl groups might not only occur for the mycotoxin $\mathrm{AOH}$, but also for AME, which differs from $\mathrm{AOH}$ by an additional methyl group (Online Resource 1). In addition, with regard to the mycotoxin AST, the exocyclic diene residue present in its structure could be a possible target for gut microbial metabolism, considering the well-known ability of the gut microbiome to reduce several functional groups, including alkenes (Koppel et al. 2017).

Anyway, as shown in Figs. 4, 5, LC-MS/MS data analysis revealed the tendency of AOH, AME, and AST to accumulate within bacterial pellets. Albeit to a much lesser extent, this phenomenon was also observed for ATX-I and ALP, while ALT, TeA, TEN, and ALS were only found in supernatants. This is in line with Król et al. (2018) who reported the ability of bacteria to bind mycotoxins.

Several parameters are considered to affect the ability of compounds to penetrate the bacterial cell walls (Macielag 2012). Among these, the $\log \mathrm{P}_{\mathrm{o} / \mathrm{w}}$ value, which is a measure of lipophilicity of molecules, plays an important role because compounds having high hydrophilicity are excluded from the passive passage through the lipid bilayer (Fost and John 1997; Macielag 2012). In the present work, a direct relation between mycotoxin concentrations in pellets and their theoretical $\log \mathrm{P}$ values was observed (Fig. 5b). The accumulation of mycotoxins in or on bacterial cells was most pronounced for AST (up to $84.9 \%$ in relation to the total recovered amount, in $P$. distasonis; $\log P=3.58 \pm 1.24$ ), followed by AME (70.57\%, E.coli; $\log P=2.55 \pm 0.62)$, and $\mathrm{AOH}(47.91 \%$, B. eggerthii; $\log P=2.17 \pm 0.60)$. The mycotoxins ATX-I and ALP accumulated to considerably less amounts, which was accompanied with low $\log P$ values $(1.64 \pm 0.77$ and $1.53 \pm 0.66$, respectively). The theoretical $\log P$ values of the other Alternaria mycotoxins not found in bacterial pellets were all below these values, except for ALS whose $\log P$ was between those of AOH and ATX-I (Fig. 5b).

Although in the present study, both Gram staining types were able to adsorb AOH, AME, and AST, Gram-negative bacteria showed higher adsorptive capacities (Fig. 5b). This tendency was not observed for ATX-I and ALP, which were present in small comparable amounts within both Gram-negative and Gram-positive bacteria. Of note, 
the preferential accumulation of AOH, AME, and AST into Gram-negative bacterial pellets was also observed after normalization of data based on a theoretical OD600 value of 0.5 (Online Resource 8). However, differences in terms of mycotoxin concentrations in pellets between the experimental and normalized data were observed and, in some cases, data normalization resulted in apparently inconsistent results such as apparent accumulation levels exceeding $100 \%$. This can be attributed to the mathematical procedure followed during the calculation which does not take into account the possible existence of a non-linear correlation between the amount of bacteria and the level of adsorbed mycotoxins due to achievement of saturation conditions or depletion of adsorbable mycotoxins. Anyway, the greater tendency of Gram-negative bacteria to accumulate $\mathrm{AOH}$ was also reported by Lemke et al. (2016), while no studies are currently available about the other Alternaria mycotoxins. The different levels of mycotoxin accumulation between the two Gram staining types might be explained by the different structure of the bacterial cell walls. Gram-negative bacteria possess a lipopolysaccharide-coated outer membrane and an inner cytoplasmic cell membrane which are separated by a thin layer of peptidoglycan. On the contrary, the cell wall surrounding Gram-positive bacteria is composed by a thick layer of peptidoglycan, but lacks the outer membrane (Denyer and Maillard 2002). Thus, the high lipid content of the Gram-negative cell wall might have favored the accumulation of the mycotoxins showing the highest log $\mathrm{P}$ values. However, considering that the use of LC-MS/ MS analysis is not suitable to determine the exact location of compounds within bacterial cells, further studies are required to elucidate this point. Interestingly, the ability of some Alternaria mycotoxins (i.e. ATX-II and AOH) to interact with eukaryotic cell membranes has already been reported (Del Favero et al. 2018,2020a,b). In particular, the mycotoxin $\mathrm{AOH}$, which is structurally similar to cholesterol, was found to affect the membrane fluidity of THP-1 macrophages and to intercalate in cholesterol-rich membrane domains (Del Favero et al. 2020b). Although sterols are not typically found in bacterial membranes and bacteria are unable to synthesize cholesterol (Huang and London 2016), this sterol was previously found in membranes of strains belonging to the genera Mycoplasma, Ehrlichia, Anaplasm, Brachyspira, Helicobacter and Borrelia, probably due to the ability of these strains to acquire cholesterol from cell hosts (Huang and London 2016). In addition, the bacterial hopanoids, which are structurally and functionally similar to sterols, were found to interact with glycolipids in bacterial outer membrane. All these findings support the possibility that some of the Alternaria mycotoxins contained in the extract were accumulated in the bacterial lipid bilayer (Sáenz et al. 2015).

\section{Conclusion}

This is the first in vitro study investigating the bidirectional relationship between the emerging class of Alternaria mycotoxins and human gut bacterial strains. Results obtained show that these potential food contaminants possess the ability to affect the growth of human gut bacterial strains and their ability to produce biofilms, suggesting that they may impair the composition and activity of the complex microbial community inhabiting human intestine. Importantly, human gut-derived microbial strains were found to adsorb some Alternaria toxins, thus indicating an important role of the gut microbiome in modulating the toxicity of these mycotoxins in humans. Taken together, this study highlights the potential role of mycotoxins in impacting gut microbiota and, vice versa, the importance of the latter in mediating the systemic bioavailability and detoxification of these abundant food contaminants consumers are constantly exposed to. Considering the well-known relation between gut microbiota and human health, subsequent in vivo studies are required to better characterize the actual risk related to this class of food contaminants. In addition, the incomplete recovery of some Alternaria mycotoxins poses the question whether gut microorganisms may transform these mycotoxins into unknown metabolites, which might also retain a certain degree of toxicity. To address this question, further studies based on untargeted high-resolution MS approaches, are first of all needed to clarify the fate of these fungal metabolites.

Supplementary Information The online version contains supplementary material available at https://doi.org/10.1007/s00204-021-03043-x.

Acknowledgments We gratefully acknowledge Fondazione Cariparma for the support given under the TeachInParma Project to F.C. and D.M., the European Research Council (Starting Grant: FunKeyGut 741623), and the University of Vienna for funding the studies. Furthermore, we thank the Mass Spectrometry Core Facility (MSC) of the University of Vienna for their support, and Dr. Alessandra Riva (University of Vienna) for providing some of the bacterial strains used in the present study.

Funding Open access funding provided by University of Vienna.

\section{Declarations}

Conflict of interest The authors declare no conflict of interest.

Availability of data and material The dataset generated during the current study is available from the corresponding author on reasonable request.

Open Access This article is licensed under a Creative Commons Attribution 4.0 International License, which permits use, sharing, adaptation, distribution and reproduction in any medium or format, as long as you give appropriate credit to the original author(s) and the source, provide a link to the Creative Commons licence, and indicate if changes were made. The images or other third party material in this article are 
included in the article's Creative Commons licence, unless indicated otherwise in a credit line to the material. If material is not included in the article's Creative Commons licence and your intended use is not permitted by statutory regulation or exceeds the permitted use, you will need to obtain permission directly from the copyright holder. To view a copy of this licence, visit http://creativecommons.org/licenses/by/4.0/.

\section{References}

Aichinger G, Krüger F, Puntscher H et al (2019) Naturally occurring mixtures of Alternaria toxins: anti-estrogenic and genotoxic effects in vitro. Arch Toxicol 93:3021-3031. https://doi.org/10. 1007/s00204-019-02545-z

Baines D, Sumarah M, Kuldau G et al (2013) Aflatoxin, fumonisin and Shiga toxin-producing Escherichia coli infections in calves and the effectiveness of celmanax ${ }^{\circledR} /$ dairyman's choice ${ }^{\mathrm{TM}}$ applications to eliminate morbidity and mortality losses. Toxins (Basel) 5:1872-1895. https://doi.org/10.3390/toxins5101872

Berthiller F, Crews C, Dall'Asta C, et al (2013) Masked mycotoxins: A review. Mol Nutr Food Res 57:165-186. https://doi.org/10.1002/ mnfr.201100764

Brugger EM, Wagner J, Schumacher DM et al (2006) Mutagenicity of the mycotoxin alternariol in cultured mammalian cells. Toxicol Lett 164:221-230. https://doi.org/10.1016/j.toxlet.2006.01.001

Burel C, Tanguy M, Guerre P et al (2013) Effect of low dose of fumonisins on pig health: immune status, intestinal microbiota and sensitivity to salmonella. Toxins (Basel) 5:841-864. https://doi.org/ 10.3390/toxins5040841

Buret AG, Motta JP, Allain T et al (2019) Pathobiont release from dysbiotic gut microbiota biofilms in intestinal inflammatory diseases: a role for iron? J Biomed Sci 26:1-14. https://doi.org/10. 1186/s12929-018-0495-4

Carding S, Verbeke K, Vipond DT et al (2015) Dysbiosis of the gut microbiota in disease. Microb Ecol Heal Dis 26(1):26191. https:// doi.org/10.3402/mehd.v26.26191

Clemente JC, Ursell LK, Parfrey LW, Knight R (2012) The impact of gut microbiota on human health: an integrative view. Cell 148:1258-1270. https://doi.org/10.1016/j.cell.2012.01.035

Collins SL, Patterson AD (2020) The gut microbiome: an orchestrator of xenobiotic metabolism. Acta Pharm Sin B 10:19-32. https:// doi.org/10.1016/j.apsb.2019.12.001

Crudo F, Varga E, Aichinger G et al (2019) Co-occurrence and combinatory effects of Alternaria mycotoxins and other xenobiotics of food origin: current scenario and future perspectives. Toxins (Basel) 11:640. https://doi.org/10.3390/toxins11110640

Crudo F, Aichinger G, Mihajlovic J et al (2020) Gut microbiota and undigested food constituents modify toxin composition and suppress the genotoxicity of a naturally occurring mixture of Alternaria toxins in vitro. Arch Toxicol 94:3541-3552. https://doi.org/ 10.1007/s00204-020-02831-1

Crudo F, Barilli A, Mena P et al (2021) An in vitro study on the transport and phase II metabolism of the mycotoxin alternariol in combination with the structurally related gut microbial metabolite urolithin C. Toxicol Lett 340:15-22. https://doi.org/10.1016/j. toxlet.2021.01.007

Del Favero G, Zaharescu R, Marko D (2018) Functional impairment triggered by altertoxin II (ATXII) in intestinal cells in vitro: crosstalk between cytotoxicity and mechanotransduction. Arch Toxicol 92:3535-3547. https://doi.org/10.1007/s00204-018-2317-6

Del Favero G, Hohenbichler J, Mayer RM et al (2020a) Mycotoxin altertoxin II induces lipid peroxidation connecting mitochondrial stress response to NF- $\mathrm{KB}$ inhibition in THP-1 macrophages. Chem Res Toxicol 33:492-504. https://doi.org/10.1021/acs.chemrestox. $9 \mathrm{~b} 00378$
Del Favero G, Mayer RM, Dellafiora L et al (2020b) Structural similarity with cholesterol reveals crucial insights into mechanisms sustaining the immunomodulatory activity of the mycotoxin alternariol. Cells 9:847. https://doi.org/10.3390/cells9040847

Dellafiora L, Warth B, Schmidt V et al (2018) An integrated in silico/in vitro approach to assess the xenoestrogenic potential of Alternaria mycotoxins and metabolites. Food Chem 248:253-261. https:// doi.org/10.1016/j.foodchem.2017.12.013

Denyer AP, Maillard JY (2002) Cellular impermeability and uptake of biocides and antibiotics in Gram-positive bacteria and mycobacteria. J Appl Microbiol Symp Suppl 92:35S-45S. https://doi.org/ 10.1046/j.1365-2672.92.5s 1.7.x

Derrien M, Belzer C, de Vos WM (2017) Akkermansia muciniphila and its role in regulating host functions. Microb Pathog 106:171-181. https://doi.org/10.1016/j.micpath.2016.02.005

Drejer Storm IML, Rasmussen RR, Rasmussen PH (2014) Occurrence of pre- and post-harvest mycotoxins and other secondary metabolites in danish maize silage. Toxins (Basel) 6:2256-2269. https:// doi.org/10.3390/toxins6082256

Espín JC, Larrosa M, García-Conesa MT, Tomás-Barberán F (2013) Biological significance of urolithins, the gut microbial ellagic acid-derived metabolites: the evidence so far. Evid Based Complement Altern Med 2013:270418. https://doi.org/10.1155/2013/ 270418

Fehr M, Pahlke G, Fritz J et al (2009) Alternariol acts as a topoisomerase poison, preferentially affecting the II $\alpha$ isoform. Mol Nutr Food Res 53:441-451. https://doi.org/10.1002/mnfr.200700379

Fleck SC, Pfeiffer E, Metzler M (2014a) Permeation and metabolism of Alternaria mycotoxins with perylene quinone structure in cultured Caco-2 cells. Mycotoxin Res 30:17-23. https://doi.org/10.1007/ s12550-013-0180-0

Fleck SC, Pfeiffer E, Podlech J, Metzler M (2014b) Epoxide reduction to an alcohol: A novel metabolic pathway for perylene quinonetype Alternaria mycotoxins in mammalian cells. Chem Res Toxicol 27:247-253. https://doi.org/10.1021/tx400366w

Fleck SC, Sauter F, Pfeiffer E et al (2016) DNA damage and repair kinetics of the Alternaria mycotoxins alternariol, altertoxin II and stemphyltoxin III in cultured cells. Mutat Res Genet Toxicol Environ Mutagen 798-799:27-34. https://doi.org/10.1016/j.mrgen tox.2016.02.001

Fost DL, John IY (1997) Biomimetic phospholipids: components. Preservative-free and self-preserving cosmetics and drugs: principles and practices. CRC Press, Boca Raton, pp 139-158

Freeman GG (1966) Isolation of alternariol and alternariol monomethyl ether from Alternaria dauci (kühn) groves and skolko. Phytochemistry 5:719-725. https://doi.org/10.1016/S0031-9422(00) 83652-3

Gambacorta L, Magistá D, Perrone G et al (2018) Co-occurrence of toxigenic moulds, aflatoxins, ochratoxin A, Fusarium and Alternaria mycotoxins in fresh sweet peppers (Capsicum annuum) and their processed products. World Mycotoxin J 11:159-173. https:// doi.org/10.3920/WMJ2017.2271

Gratz SW, Dinesh R, Yoshinari T et al (2017) Masked trichothecene and zearalenone mycotoxins withstand digestion and absorption in the upper GI tract but are efficiently hydrolyzed by human gut microbiota in vitro. Mol Nutr Food Res 61:11-24. https://doi.org/ $10.1002 / \mathrm{mnfr} .201600680$

Grover S, Lawrence CB (2017) The Alternaria alternata mycotoxin alternariol suppresses lipopolysaccharide-induced inflammation. Int J Mol Sci 18:1577. https://doi.org/10.3390/ijms18071577

Guo M, Huang K, Chen S et al (2014) Combination of metagenomics and culture-based methods to study the interaction between ochratoxin a and gut microbiota. Toxicol Sci 141:314-323. https://doi. org/10.1093/toxsci/kfu128

Haney EF, Trimble MJ, Cheng JT et al (2018) Critical assessment of methods to quantify biofilm growth and evaluate antibiofilm 
activity of host defence peptides. Biomolecules 8:1-22. https:// doi.org/10.3390/biom8020029

Hasan N, Yang H (2019) Factors affecting the composition of the gut microbiota, and its modulation. PeerJ 2019:1-31. https://doi.org/ $10.7717 /$ peerj. 7502

He P, Young LG, Forsberg C (1992) Microbial transformation of deoxynivalenol (vomitoxin). Appl Environ Microbiol 58:3857-3863. https://doi.org/10.1128/aem.58.12.3857-3863.1992

Hellwig V, Grothe T, Mayer-Bartschmid A et al (2002) Altersetin, a new antibiotic from cultures of endophytic Alternaria spp. Taxonomy, fermentation, isolation, structure elucidation and biological activities. J Antibiot (Tokyo) 55:881-892. https:// doi.org/10.7164/antibiotics.55.881

Huang Z, London E (2016) Cholesterol lipids and cholesterol-containing lipid rafts in bacteria. Chem Phys Lipids 199:11-16. https://doi.org/10.1016/j.chemphyslip.2016.03.002

Jandhyala SM, Talukdar R, Subramanyam C et al (2015) Role of the normal gut microbiota. World J Gastroenterol 21:8836-8847. https://doi.org/10.3748/wjg.v21.i29.8787

Jarolim K, Del Favero G, Ellmer D et al (2017) Dual effectiveness of Alternaria but not Fusarium mycotoxins against human topoisomerase II and bacterial gyrase. Arch Toxicol 91:2007-2016. https://doi.org/10.1007/s00204-016-1855-z

Kjer J, Wray V, Edrada-Ebel RA et al (2009) Xanalteric acids I and II and related phenolic compounds from an endophytic Alternaria sp. isolated from the Mangrove plant Sonneratia alba. J Nat Prod 72:2053-2057. https://doi.org/10.1021/np900417g

Kollarczik B, Gareis M, Hanelt M (1994) In vitro transformation of the Fusarium mycotoxins deoxynivalenol and zearalenone by the normal gut microflora of pigs. Nat Toxins 2:105-110. https://doi.org/10.1002/nt.2620020303

Kollarova J, Cenk E, Schmutz C, Marko D (2018) The mycotoxin alternariol suppresses lipopolysaccharide-induced inflammation in THP- 1 derived macrophages targeting the NF- $\mathrm{KB}$ signalling pathway. Arch Toxicol 92:3347-3358. https://doi.org/10.1007/ s00204-018-2299-4

Koppel N, Rekdal VM, Balskus EP (2017) Chemical transformation of xenobiotics by the human gut microbiota. Science 356:12461257. https://doi.org/10.1126/science.aag2770

Król A, Pomastowski P, Rafińska K et al (2018) Microbiology neutralization of zearalenone using Lactococcus lactis and Bifidobacterium sp. Anal Bioanal Chem 410:943-952. https://doi.org/ 10.1007/s00216-017-0555-8

Lee JH, Kim YG, Cho HS et al (2014) Coumarins reduce biofilm formation and the virulence of Escherichia coli O157:H7. Phytomedicine 21:1037-1042. https://doi.org/10.1016/j.phymed. 2014.04.008

Lehmann L, Wagner J, Metzler M (2006) Estrogenic and clastogenic potential of the mycotoxin alternariol in cultured mammalian cells. Food Chem Toxicol 44:398-408. https://doi.org/ 10.1016/j.fct.2005.08.013

Lemke A, Burkhardt B, Bunzel D et al (2016) Alternaria toxins of the alternariol type are not metabolised by human faecal microbiota. World Mycotoxin J 9:41-49. https://doi.org/10. 3920/WMJ2014.1875

Liew WPP, Mohd-Redzwan S (2018) Mycotoxin: Its impact on gut health and microbiota. Front Cell Infect Microbiol 8:60. https:// doi.org/10.3389/fcimb.2018.00060

Lou J, Yu R, Wang X et al (2016) Alternariol 9-methyl ether from the endophytic fungus Alternaria sp. Samif01 and its bioactivities. Braz J Microbiol 47:96-101. https://doi.org/10.1016/j.bjm. 2015.11.004

Macielag MJ (2012) Chemical properties of antimicrobials and their uniqueness. In: Dougherty TJ, Pucci M (eds) Antibiotic discovery and development. Springer, Boston, pp 793-820
Majidpour A, Fathizadeh S, Afshar M et al (2017) Dose-dependent effects of common antibiotics used to treat Staphylococcus aureus on biofilm formation. Iran J Pathol 12:362-370. https:// doi.org/10.30699/ijp.2017.27993

Makarewicz M, Drożdż I, Tarko T, Duda-Chodak A (2021) The Interactions between polyphenols and microorganisms, especially gut microbiota. Antioxidants 10:188. https://doi.org/10. 3390/antiox 10020188

Ouethrani M, Van De WT, Verbeke E et al (2013) Metabolic fate of ochratoxin A as a coffee contaminant in a dynamic simulator of the human colon. Food Chem 141:3291-3300. https://doi.org/ 10.1016/j.foodchem.2013.05.157

Oviedo MS, Barros GG, Chulze SN, Ramirez ML (2012) Natural occurrence of alternariol and alternariol monomethyl ether in soya beans. Mycotoxin Res 28:169-174. https://doi.org/10. 1007/s12550-012-0132-0

Papa R, Garzoli S, Vrenna G et al (2020) Essential oils biofilm modulation activity, chemical and machine learning analysis. Application on Staphylococcus aureus isolates from cystic fibrosis patients. Int J Mol Sci 21:1-20. https://doi.org/10.3390/ijms2 1239258

Perna A, Hay E, Contieri M et al (2020) Adherent-invasive Escherichia coli (AIEC): cause or consequence of inflammation, dysbiosis, and rupture of cellular joints in patients with IBD? J Cell Physiol 235:5041-5049. https://doi.org/10.1002/jcp.29430

Pero RW, Owens RG, Dale SW, Harvan D (1971) Isolation and indentification of a new toxin, altenuene, from the fungus Alternaria tenuis. Biochim Biophys Acta 230:170-179. https://doi. org/10.1016/0304-4165(71)90064-X

Piotrowska M, Ślizewska K, Nowak A et al (2014) The effect of experimental Fusarium mycotoxicosis on microbiota diversity in porcine ascending colon contents. Toxins (Basel) 6:20642081. https://doi.org/10.3390/toxins6072064

Puntscher H, Kütt ML, Skrinjar P et al (2018) Tracking emerging mycotoxins in food: development of an LC-MS/MS method for free and modified Alternaria toxins. Anal Bioanal Chem 410:4481-4494. https://doi.org/10.1007/s00216-018-1105-8

Puntscher H, Aichinger G, Grabher S et al (2019a) Bioavailability, metabolism, and excretion of a complex Alternaria culture extract versus altertoxin II: a comparative study in rats. Arch Toxicol 93:3153-3167. https://doi.org/10.1007/ s00204-019-02575-7

Puntscher H, Hankele S, Tillmann K et al (2019b) First insights into Alternaria multi-toxin in vivo metabolism. Toxicol Lett 301:168178. https://doi.org/10.1016/j.toxlet.2018.10.006

Righetti L, Paglia G, Galaverna G, Dall'Asta C (2016) Recent advances and future challenges in modified mycotoxin analysis: why HRMS has become a key instrument in food contaminant research. Toxins (Basel) 8(12):361. https://doi.org/10.3390/toxins8120361

Sáenz JP, Grosser D, Bradley AS et al (2015) Hopanoids as functional analogues of cholesterol in bacterial membranes. Proc Natl Acad Sci USA 112:11971-11976. https://doi.org/10.1073/pnas.15156 07112

Saint-Cyr MJ, Perrin-Guyomard A, Houée P et al (2013) Evaluation of an oral subchronic exposure of deoxynivalenol on the composition of human gut microbiota in a model of human microbiotaassociated rats. PLoS ONE 8(11):e80578. https://doi.org/10.1371/ journal.pone.0080578

Schiller C, Fröhlich CP, Giessmann T et al (2005) Intestinal fluid volumes and transit of dosage forms as assessed by magnetic resonance imaging. Aliment Pharmacol Ther 22:971-979. https://doi. org/10.1111/j.1365-2036.2005.02683.x

Schuchardt S, Ziemann C, Hansen T (2017) Combined toxicokinetic and in vivo genotoxicity study on Alternaria toxins. EFSA Support Publ 11(11):679E. https://doi.org/10.2903/sp.efsa.2014. en-679 
Scott KP, Gratz SW, Sheridan PO et al (2013) The influence of diet on the gut microbiota. Pharmacol Res 69:52-60. https://doi.org/10. 1016/j.phrs.2012.10.020

Stepanović S, Vuković D, Hola V et al (2007) Quantification of biofilm in microtiter plates: overview of testing conditions and practical recommendations for assessment of biofilm production by staphylococci. APMIS 115:891-899. https://doi.org/10.1111/j. 1600-0463.2007.apm_630.x

Stinson EE, Osman SF, Heisler EG et al (1981) Mycotoxin production in whole tomatoes, apples, oranges, and lemons. J Agric Food Chem 29:790-792. https://doi.org/10.1021/jf00106a025

Tenk I, Fodor E, Szathmary C (1982) The effect of pure Fusarium toxins (T-2, F-2, DAS) on the microflora of the gut and on plasma glucocorticoid levels in rat and swine. Zentralblatt fur Bakteriol Mikrobiol und Hyg 252:384-393. https://doi.org/10.1016/s01743031(82)80013-9

Theophel K, Schacht VJ, Schlüter M et al (2014) The importance of growth kinetic analysis in determining bacterial susceptibility against antibiotics and silver nanoparticles. Front Microbiol 5:1-10. https://doi.org/10.3389/fmicb.2014.00544

Wang J, Tang L, Glenn TC, Wang JS (2016) Aflatoxin B1 induced compositional changes in gut microbial communities of male F344 rats. Toxicol Sci 150:54-63. https://doi.org/10.1093/toxsci/ kfv259
Worrell NR, Mallett AK, Cook WM et al (1989) The role of gut microorganisms in the metabolism of deoxynivalenol administered to rats. Xenobiotica 19:25-32. https://doi.org/10.3109/0049825890 9034673

Young JC, Zhou T, Yu H et al (2007) Degradation of trichothecene mycotoxins by chicken intestinal microbes. Food Chem Toxicol 45:136-143. https://doi.org/10.1016/j.fct.2006.07.028

$\mathrm{Yu} \mathrm{H}$, Zhou T, Gong J et al (2010) Isolation of deoxynivalenol-transforming bacteria from the chicken intestines using the approach of PCR-DGGE guided microbial selection. BMC Microbiol 10(1):19. https://doi.org/10.1186/1471-2180-10-182

Zhao K, Shao B, Yang D et al (2015) Natural occurrence of Alternaria toxins in wheat-based products and their dietary exposure in China. PLoS ONE 10:1-11. https://doi.org/10.1371/journal. pone.0132019

Zhou Y, Zhi F (2016) Lower level of Bacteroides in the gut microbiota is associated with inflammatory bowel disease: a meta-analysis. Biomed Res Int 2016:1-9. https://doi.org/10.1155/2016/5828959

Publisher's Note Springer Nature remains neutral with regard to jurisdictional claims in published maps and institutional affiliations. 